\title{
EIGENVALUES IN SPECTRAL GAPS OF A PERTURBED PERIODIC MANIFOLD
}

\author{
OLAF POST
}

\begin{abstract}
We consider a non-compact Riemannian periodic manifold such that the corresponding Laplacian has a spectral gap. By continuously perturbing the periodic metric locally we can prove the existence of eigenvalues in a gap. A lower bound on the number of eigenvalue branches crossing a fixed level is established in terms of a discrete eigenvalue problem. Furthermore, we discuss examples of perturbations leading to infinitely many eigenvalue branches coming from above resp. finitely many branches coming from below.
\end{abstract}

\section{INTRODUCTION}

A periodic manifold is a (non-compact) Riemannian manifold $\mathcal{M}$ with metric $g$ such that a finitely generated Abelian group $\Gamma$ acts properly discontinuously and isometrically on $\mathcal{M}$. Furthermore, we assume that the quotient $\mathcal{M} / \Gamma$ is compact. We call the closure of a fundamental domain a period cell. We suppose that the Laplacian $\Delta_{\mathcal{M}}$ on $\mathcal{M}$ has a spectral gap $I=(a, b)$ in the (essential) spectrum, i.e.,

$$
I \cap \operatorname{spec} \Delta_{\mathcal{M}}=\emptyset
$$

where $a>\inf$ ess spec $\Delta_{\mathcal{M}}$. We prove that a local perturbation of the periodic structure leads to eigenvalues in the gap for large deformation parameters $\tau$. More precisely, we perturb the periodic metric $g$ smoothly, starting from $g=g_{0}$ and we denote the manifold $\mathcal{M}$ with perturbed metric $g_{\tau}$ by $\mathcal{M}_{\tau}$. Furthermore, we assume that $g_{\tau}$ and its first derivatives depend continuously on $\tau$ and that the perturbation is (essentially) contained in the compact set $M^{m}$. Here, $M^{m}$ denotes a finite number of copies of a period cell $M$ of $\mathcal{M}$. Later on we allow perturbations $\mathcal{M}_{\tau}$ diffeomorphic to $\mathcal{M}$ (see Section 6) or even some cases when the perturbation changes the topology of $\mathcal{M}$ (see Section (7).

Donnelly [Do2] and Li [Li] proved the existence of eigenvalues below the essential spectrum of the Laplacian on the simply connected, complete, hyperbolic plane by a local perturbation of the manifold. The methods employed there use separation of variables. Therefore the ideas cannot be generalized to our case. Furthermore the Min-max principle applies, since the eigenvalues lie below the

Date: 05.12 .2001 .

1991 Mathematics Subject Classification. 35P20, 58G18, 47F05.

Key words and phrases. Eigenvalues, spectral gap, perturbation of periodic structures. 
essential spectrum. Note also the introductory article [Hi] concerning results on the spectral theory of hyperbolic manifolds.

In general, it is much more difficult to prove the existence of eigenvalues in a spectral gap. Such problems have been extensively studied in the case of Schrödinger or divergence type operators with a spectral gap (see e.g. [DH], [ADH], AADH] or [HB]). The school of Birman (see e.g. [Bi] or [Sa] and references given therein) obtained a vast number of results on the asymptotics of eigenvalue counting functions using the Birman-Schwinger principle. This principle does not apply to our case since the perturbation is not additive. General results on perturbation of point spectra can be found in [ [ $\mathrm{K}$ or [RS4].

From a physical point of view the Laplacian on a periodic manifold is the Hamiltonian of an electron confined to a periodically curved material, cf. [FH] or M. For example, consider a flat small strip in $\mathbb{R}^{2}$ (i.e., a quantum wire or quantum wave guide) which is periodically curved. If the strip is thin enough, there exist gaps in the spectrum of the Dirichlet Laplacian (cf. [ES] and $\square]$ ). The local perturbation of the periodic structure leads to bound states (i.e. square integrable eigenfunctions) in a spectral gap. Physically, the perturbation corresponds to local impurities coming from contamination, e.g., in a semi-conductor. In particular, we show that local deformations of a periodically curved quantum wire produce bound states with energy in a spectral gap of the Hamiltonian of the non-perturbed quantum wire (see Proposition 6).

Assumptions. The main assumption on the periodic manifold $\mathcal{M}$ resp. the period cell $M$ is the following. Let $\lambda_{k}^{\mathrm{D}}(M)$ and $\lambda_{k}^{\mathrm{N}}(M)$ denote the eigenvalues (written in increasing order and repeated according to multiplicity) of the Laplacian on the period cell $M$ with Dirichlet resp. Neumann boundary condition on $\partial M$. Note that we always have $\lambda_{k}^{\mathrm{N}}(M) \leq \lambda_{k}^{\mathrm{D}}(M)$ in virtue of the Min-max principle (see the next section for details). We assume that the following gap condition is fulfilled:

$$
\exists k \in \mathbb{N}: \quad I_{k}:=\left(\lambda_{k}^{\mathrm{D}}(M), \lambda_{k+1}^{\mathrm{N}}(M)\right) \neq \emptyset,
$$

i.e., $\lambda_{k}^{\mathrm{D}}(M)<\lambda_{k+1}^{\mathrm{N}}(M)$ for some $k$. From Floquet theory and the Min-max principle it follows that $I_{k}$ lies in a spectral gap for the Laplacian on $\mathcal{M}$. Note that $I_{k}$ could be strictly contained in a spectral gap. The gap condition (11.1) is fulfilled if the period cell decouples from its translates, i.e., if the junctions between different period cells are small in some sense. Recall that the gap condition is not satisfied for $\mathcal{M}=\mathbb{R}^{d}$ with the standard metric. In this case, we always have $\lambda_{k}^{\mathrm{D}}(M) \geq \lambda_{k+1}^{\mathrm{N}}(M)$. In Section 5 we give examples of periodic manifolds with an arbitrary (finite) number of gaps that were obtained in [P2] and [Y].

If $\Gamma^{\prime}$ denotes a subset of the Abelian group $\Gamma$ we set

$$
\Gamma^{\prime} M:=\bigcup_{\gamma \in \Gamma^{\prime}} \gamma M,
$$


in particular, $\Gamma M=\mathcal{M}$. Consider a sequence $\left(\Gamma^{n}\right)$ of subsets of $\Gamma$ such that $\Gamma^{n}$ has $n$ elements. We assume that $\Gamma^{n} \nearrow \Gamma$ and set $M^{n}:=\Gamma^{n} M, R^{m}:=\left(\Gamma \backslash \Gamma^{m}\right) M$ and $R^{m, n}:=\left(\Gamma^{n} \backslash \Gamma^{m}\right) M$. As for $\mathcal{M}_{\tau}$ we denote by $M_{\tau}^{n}$ resp. $R_{\tau}^{m, n}$ the manifold $M^{n}$ resp. $R^{m, n}$ with perturbed metric $g_{\tau}$.

The assumptions on the family $\left(g_{\tau}\right)$ of perturbed metrics on $\mathcal{M}$ are the following:

$$
\begin{aligned}
g_{0}=g & \text { on } \mathcal{M} \\
\left\|g_{\tau}-g_{\tau_{0}}\right\|_{C^{1}} \rightarrow 0 & \text { on } \mathcal{M} \text { as } \tau \rightarrow \tau_{0} \\
g_{\tau}=g_{0} & \text { on } R^{m}, \tau \geq 0
\end{aligned}
$$

for all $\tau_{0} \geq 0$ and sufficiently large $m \in \mathbb{N}$. The $C^{1}$-norm of a metric is defined in (3.2). Furthermore, if $\partial M$ is smooth we can also allow non-compact perturbations which are small outside $M^{m}$, i.e.,

$$
\sup _{\tau \geq 0}\left\|g_{\tau}-g_{0}\right\|_{C^{1}} \rightarrow 0 \quad \text { on } R^{m} \text { as } m \rightarrow \infty .
$$

Note that $\partial M$ is smooth iff $\partial R^{m}$ is smooth.

Assumption (1.2) assures that we start from the periodic manifold. Assumption (1.3) guarantees the continuous dependence of the metric and its first derivatives on $\tau$. In particular, eigenvalues on the approximating problem $M_{\tau}^{n}$ depend continuously on $\tau$ (cf. Corollary 3.10), and the norm resp. weak topologies of the corresponding Hilbert spaces do not depend on $\tau$ (cf. Corollary 3.9). Note that we only need to control the metric up to its first derivatives since no higher derivative of the metric occurs in the coefficients of the Laplacian, cf. Equation (2.1).

Finally, Assumption (1.4) resp. (1.4) says that the support of the perturbation is contained in the compact set $M^{m}$ resp. is small outside the compact set $M^{m}$. In particular, one can prove a decomposition principle for non-compact perturbations, i.e., the invariance of the essential spectrum under such perturbations (see Theorem 4.1). With other words, the essential spectrum reflects the geometry at infinity. In our situation, the decomposition principle assures that the spectral gaps of $\Delta_{\mathcal{M}}$ remain spectral gaps in the essential spectrum of $\Delta_{\mathcal{M}_{\tau}}$ for all $\tau \geq 0$. Only discrete eigenvalues (possibly accumulating at the band-edges of $\operatorname{spec} \Delta_{\mathcal{M}}$ ) can appear in the spectral gaps.

Main result. To count the number of eigenvalues $\lambda$ (with multiplicity) of the family $\Delta_{\mathcal{M}_{\tau^{\prime}}}, 0 \leq \tau^{\prime} \leq \tau$, we define

$$
\mathcal{N}(\tau, \lambda):=\sum_{0 \leq \tau^{\prime} \leq \tau} \operatorname{dim} \operatorname{ker}\left(\Delta_{\mathcal{M}_{\tau^{\prime}}}-\lambda\right)
$$

Note the difference to the ordinary eigenvalue counting function

$$
\operatorname{dim}_{I}^{\mathrm{D}}(M):=\sum_{\lambda \in I} \operatorname{dim} \operatorname{ker}\left(\Delta_{M}^{\mathrm{D}}-\lambda\right)
$$


that counts the eigenvalues in the Borel subset $I \subset \mathbb{R}_{+}$of the Dirichlet Laplacian on $M$; similarly, $\operatorname{dim}_{I}^{\mathrm{N}}(M)$ denotes the number of eigenvalues in $I$ of the Neumann Laplacian. As an abbreviation, we set $\operatorname{dim}_{\lambda}^{\mathrm{D}}(M):=\operatorname{dim}_{[0, \lambda]}^{\mathrm{D}}(M)$.

Our main result is the following:

Theorem 1.1. Let $\lambda \in I_{k}$ belong to a spectral gap of $\Delta_{\mathcal{M}}$ then

$$
\begin{aligned}
& \mathcal{N}(\tau, \lambda) \geq \operatorname{dim}_{\lambda}^{\mathrm{D}}\left(M_{\tau}^{m}\right)-\operatorname{dim}_{\lambda}^{\mathrm{D}}\left(M^{m}\right) \\
& \mathcal{N}(\tau, \lambda) \geq \operatorname{dim}_{\lambda}^{\mathrm{N}}\left(M^{m}\right)-\operatorname{dim}_{\lambda}^{\mathrm{N}}\left(M_{\tau}^{m}\right)
\end{aligned}
$$

for all $\tau \geq 0$ and sufficiently large $m \in \mathbb{N}$.

Note that Theorem 1.1 reduces the eigenvalue problem on the non-compact manifold $\mathcal{M}$ to one on the compact manifold $M^{m}$. Therefore, we can apply the Min-max principle (2.2) to assure the existence of eigenfunctions of the perturbed problem on $M_{\tau}^{m}$.

In a semi-classical picture, the number of bound states for high energy levels is approximately given by the phase space volume associated with the classical energy of the quantum system. The classical Hamiltonian on a $d$-dimensional Riemannian manifold $X$ corresponding to $\Delta_{X}$ is given by $h(x, p):=g_{x}^{*}(p, p)$ where $g^{*}$ denotes the metric on the cotangent bundle $T^{*} X$ and $p \in T_{x}^{*} X$. The Hamiltonian $h$ determines a region in the phase space $T^{*} X$ in which a classical particle with given energy is allowed to move. The uncertainty principle, however, demands that each bound state requires a cube of volume $(2 \pi)^{d}$ in phase space, and therefore the total number of bound states is approximately equal to the phase space volume divided by $(2 \pi)^{d}$ (see [RS4]). Indeed, the Weyl asymptotic distribution of the (Dirichlet or Neumann) eigenvalues of the Laplacian on $X$ is given by

$$
\operatorname{dim}_{\lambda}(X)-\frac{\omega_{d}}{(2 \pi)^{d}} \lambda^{\frac{d}{2}} \operatorname{vol}(X)=O\left(\lambda^{\frac{d-1}{2}}\right), \quad \lambda \rightarrow \infty
$$

where $\omega_{d}$ is the volume of the unit ball in $\mathbb{R}^{d}$ (see e.g. [SV]). Since we expect that the bound states are localized on the perturbed manifold $M^{m}$ we anticipate that

$$
\frac{\omega_{d}}{(2 \pi)^{d}} \lambda^{\frac{d}{2}}\left|\operatorname{vol}\left(M^{m}\right)-\operatorname{vol}\left(M_{\tau}^{m}\right)\right|
$$

is a lower bound for the eigenvalue counting function (1.5). However, note that $O\left(\lambda^{\frac{d-1}{2}}\right)$ in (1.8) depends on the manifold $M^{m}$. Nevertheless in our examples in Sections 6 and 7 we show that an infinite number of eigenvalue branches crosses the level $\lambda$ from above if the volume of $M_{\tau}^{m}$ increases. In contrast, if we decrease the volume of $M_{\tau}^{m}$, only a finite number of eigenvalue branches cross the level $\lambda$ from below. Here, by an eigenvalue branch we mean an eigenvalue of (one of) the perturbed manifold(s) viewed as a function depending on the perturbation parameter $\tau$.

Under the assumptions given above it is not possible to obtain an upper bound on the function $\mathcal{N}(\tau, \lambda)$. Since the dependence on $\tau$ is not supposed to be analytic 
in Assumption (1.3), we could consider a family of metrics $\left(g_{\tau}\right)$ which is constant near $\tau_{0}$. If $\lambda$ is an eigenvalue of $\Delta_{\mathcal{M}_{\tau_{0}}}$, then $\mathcal{N}\left(\tau_{0}, \lambda\right)=\infty$.

Nevertheless, the examples in Sections 6 and 7 in which the eigenvalue branches of the problem on $M_{\tau}^{m}$ are monotonical should be good candidates where an upper bound on the counting function $\mathcal{N}(\tau, \lambda)$ in terms of $\left|\operatorname{dim}_{\lambda}\left(M_{\tau}^{m}\right)-\operatorname{dim}_{\lambda}\left(M^{m}\right)\right|$ holds (cf. Remarks 6.1, 6.3 and 7.3). Under suitable further conditions one should have to assure that the eigenvalue branches (in the gap) of the full problem $\mathcal{M}_{\tau}$ are strictly monotonical. Note that - in contrast to the approximating problem on $M_{\tau}^{m}$ - the monotonicity of the eigenvalue branches of the full problem $\mathcal{M}_{\tau}$ cannot be shown by the Min-max principle since the gap lies above the infimum of the essential spectrum.

Note furthermore that the counting function defined above could be much greater than the spectral flow of the family $\left(\Delta_{M_{\tau}}\right)$ at $\lambda$ defined as the difference of the eigenvalue branches crossing the level $\lambda$ from above and below (cf. e.g. Sa $)$. Think for example of an eigenvalue branch which oscillates around $\lambda$.

In [DH or $\mathrm{ADH})$ a family $\left(\Delta_{\mathcal{M}_{\tau}}\right)$ is called complete if for each $\lambda \geq 0$ there exists $\tau \geq 0$ such that $\lambda \in \operatorname{spec} \Delta_{\mathcal{M}_{\tau}}$. Note that all our examples given in Sections 6 and 7 are complete in this sense (in Proposition 6 we have to perturb at least $m=2$ period cells to ensure that $\mathcal{N}(\tau, \lambda) \geq 1$ for $\tau$ large enough).

For the proof of Theorem 1.1 we cannot directly apply the Min-max principle since we consider eigenvalues inside a spectral gap. Therefore, we adopt the following idea given in [DH], [ADH], AADH or [HB to our situation: we show that the eigenfunctions of the full problem on $\mathcal{M}$ can be approximated by eigenfunctions of an approximating problem on $M^{n}$ (cf. Theorem 4.3 and Theorem 4.5). Our situation differs from the case given in the papers mentioned above: in some sense it is more complicated since we deal with different Hilbert spaces $L_{2}\left(\mathcal{M}_{\tau}\right)$ for each $\tau$. On the other hand, our situation is simpler since there are no extra eigenvalues in the gap coming from the boundary conditions on the approximating problem (cf. Lemma 4.2).

The paper is organized as follows: In Section 2 we provide some notation and basic results on Laplacians and periodic manifolds. In Section 3 we develop elliptic estimates needed for the proof of Theorem 1.1 given in Section 1 . In Section 5 we recall examples of periodic manifolds with spectral gaps. Finally, in Sections 6 and 7 we present examples of diffeomorphic and non-homeomorphic perturbations leading to eigenvalues.

\section{Preliminaries}

Laplacian on a manifold. Throughout this article we study manifolds of dimension $d \geq 2$. For a Riemannian manifold $M$ (compact or not) without boundary we denote by $L_{2}(M)$ the usual $L_{2}$-space of square integrable functions on $M$ with respect to the volume measure on $M$. In a chart, the volume measure has the density $(\operatorname{det} g)^{\frac{1}{2}}$ with respect to the Lebesgue measure, where $\operatorname{det} g$ is the 
determinant of the metric tensor $\left(g_{i j}\right)$ in this chart. The norm of $L_{2}(M)$ will be denoted by $\|\cdot\|_{M}$. For $u \in C_{\mathrm{c}}^{\infty}(M)$, the space of compactly supported smooth functions, we set

$$
\check{q}_{M}(u):=\|\mathrm{d} u\|_{M}^{2}=\int_{M}|\mathrm{~d} u|^{2} .
$$

Here the 1-form $\mathrm{d} u$ denotes the exterior derivative of $u$ given in coordinates by $|\mathrm{d} u|^{2}=\sum_{i, j} g^{i j} \partial_{i} u \partial_{j} \bar{u}$ where $\left(g^{i j}\right)$ is the inverse matrix of $\left(g_{i j}\right)$.

We denote the closure of the non-negative quadratic form $\check{q}_{M}$ by $q_{M}$. Note that the domain dom $q_{M}$ of the closed quadratic form $q_{M}$ consists of functions in $L_{2}(M)$ such that the weak derivative $\mathrm{d} u$ is also square integrable (i.e., $q_{M}(u)<\infty$ ).

We define the Laplacian $\Delta_{M}$ (for a manifold without boundary) as the unique self-adjoint and non-negative operator associated with the closed quadratic form $q_{M}$, i.e., operator and quadratic form are related by

$$
q_{M}(u)=\left\langle\Delta_{M} u, u\right\rangle
$$

for $u \in C_{\mathrm{c}}^{\infty}(M)$ (for details on quadratic forms see e.g. [K, Chapter VI], [RS1] or [Da]). Therefore, the Laplacian for smooth functions $u$ is given in a chart by

$$
\Delta_{M} u=-(\operatorname{det} g)^{-\frac{1}{2}} \sum_{i, j} \partial_{i}\left((\operatorname{det} g)^{\frac{1}{2}} g^{i j} \partial_{j} u\right) .
$$

If $M$ is a complete manifold with piecewise smooth boundary $\partial M \neq \emptyset$ we can define the Laplacian with Dirichlet resp. Neumann boundary condition in the same way. Here, we start from the (closure of the) quadratic form $\check{q}_{M}$ defined on $C_{\mathrm{c}}^{\infty}(M)$, the space of smooth functions with compact support away from the boundary, resp. on the subspace of $C^{\infty}(M)$ with $\|u\|_{M}^{2}$ and $q_{M}(u)$ finite. Here, $C^{\infty}(M)$ denotes the space of smooth functions with derivatives continuous up to the boundary of $M$. We denote the closure of the quadratic form by $q_{M}^{\mathrm{D}}$ resp. $q_{M}^{\mathrm{N}}$ and the corresponding self-adjoint operator by $\Delta_{M}^{\mathrm{D}}$ resp. $\Delta_{M}^{\mathrm{N}}$. If we are only interested in the differential expression of the Laplacian, we suppress the boundary condition label. If $\partial M$ can be split into two disjoint closed sets $\partial_{1} M$ and $\partial_{2} M$ we define the Laplacian with mixed boundary conditions,. i.e., Dirichlet boundary condition on $\partial_{1} M$ and Neumann boundary condition on $\partial_{2} M$ in the obvious way.

If $M$ is compact the spectrum of $\Delta_{M}$ (with any boundary condition if $\partial M \neq \emptyset$ ) is purely discrete. Note that we always assume that $\partial M$ is piecewise smooth. We denote the corresponding eigenvalues by $\lambda_{k}(M), k \in \mathbb{N}$, (resp. $\lambda_{k}^{\mathrm{D}}(M)$ or $\lambda_{k}^{\mathrm{N}}(M)$ in the Dirichlet or Neumann case) written in increasing order and repeated according to multiplicity. The corresponding eigenfunctions are $C^{\infty}(M)$ up to the boundary. With this eigenvalue ordering, we can state the Min-max principle (for this version of the Min-max principle see e.g. [Da]). The $k$-th eigenvalue of 
$\Delta_{M}^{\mathrm{D}}$ can be expressed by

$$
\lambda_{k}^{\mathrm{D}}(M)=\inf _{L_{k}} \sup _{u \in L_{k}, u \neq 0} \frac{\|\mathrm{d} u\|^{2}}{\|u\|^{2}}
$$

where the infimum is taken over all $k$-dimensional subspaces $L_{k}$ of $\operatorname{dom} q_{M}^{\mathrm{D}}$. Similar results hold for Neumann or other boundary conditions.

Suppose now that $M=M_{1} \cup M_{2}$ such that $M_{1}$ and $M_{2}$ have piecewise smooth boundary and that $M_{1} \cap M_{2}$ has measure 0 . Denote by $\lambda_{k}^{\mathrm{N}}\left(M_{1} \dot{\cup} M_{2}\right)$ the eigenvalues of the quadratic form $q_{M_{1}}^{\mathrm{N}} \oplus q_{M_{2}}^{\mathrm{N}}$ and similarly for Dirichlet boundary condition. Then the Min-max principle implies

$$
\lambda_{k}^{\mathrm{N}}\left(M_{1} \dot{\cup} M_{2}\right) \leq \lambda_{k}^{\mathrm{N}}(M) \leq \lambda_{k}^{\mathrm{D}}(M) \leq \lambda_{k}^{\mathrm{D}}\left(M_{1} \dot{\cup} M_{2}\right)
$$

for all $k \in \mathbb{N}$ since the opposite inclusions hold for the corresponding quadratic form domains. This estimate is called Dirichlet-Neumann bracketing.

Periodic manifolds and Floquet Theory. Let $\Gamma$ be an Abelian group of infinite order with $r$ generators and neutral element 1 . Such groups are isomorphic to $\mathbb{Z}^{r_{0}} \times \mathbb{Z}_{p_{1}}^{r_{1}} \times \cdots \times \mathbb{Z}_{p_{a}}^{r_{a}}$ with $r_{0}>0$ and $r_{0}+\cdots+r_{a}=r$. Here, $\mathbb{Z}_{p}$ denotes the Abelian group of order $p$. A $d$-dimensional (non-compact) Riemannian manifold $\mathcal{M}$ will be called periodic (or covering manifold) if $\Gamma$ acts properly discontinuously, isometrically and cocompactly on $\mathcal{M}$. Cocompactness means that the quotient space $\mathcal{M} / \Gamma$ is compact. Note that the quotient space is a $d$-dimensional compact Riemannian manifold and that the quotient map $\pi: \mathcal{M} \longrightarrow \mathcal{M} / \Gamma$ is a local isometry. The metric turning the manifold $\mathcal{M}$ into a periodic one will also be called periodic. For details on periodic manifolds see e.g. [C2.

A compact subset $M$ of $\mathcal{M}$ is called a period cell if $M$ is the closure of a fundamental domain $D$, i.e., $M=\bar{D}, D$ is open and connected, $D$ is disjoint from any translate $\gamma D$ for all $\gamma \in \Gamma, \gamma \neq 1$, and the union of all translates $\gamma M$ is equal to $\mathcal{M}$. Furthermore we assume that $M$ has piecewise smooth boundary.

Floquet theory allows us to analyse the spectrum of the Laplacian on $\mathcal{M}$ by analysing the spectra of Laplacians with quasi-periodic boundary condition on a period cell $M$. In order to do this, we define $\theta$-periodic boundary condition. Let $\theta$ be an element of the dual group $\hat{\Gamma}=\operatorname{Hom}\left(\Gamma, \mathbb{T}^{1}\right)$ of $\Gamma$, which is isomorphic to a closed subgroup of the $r$-dimensional torus $\mathbb{T}^{r}=\left\{\theta \in \mathbb{C}^{r} ;\left|\theta_{i}\right|=1\right.$ for all $\left.i\right\}$. We denote by $q_{M}^{\theta}$ the closure of the quadratic form $\check{q}_{M}$ defined on the space of those functions $u \in C^{\infty}(M)$ that satisfy

$$
u(\gamma x)=\overline{\theta(\gamma)} u(x)
$$

for all $x \in \partial M$ and all $\gamma \in \Gamma$ such that $\gamma x \in \partial M$. The corresponding operator is denoted by $\Delta_{M}^{\theta}$. Again, $\Delta_{M}^{\theta}$ has purely discrete spectrum denoted by $\lambda_{k}^{\theta}(M)$. The eigenvalues depend continuously on $\theta$ (see [RS4] or [BJR]). From Floquet 
theory we obtain

$$
\operatorname{spec} \Delta_{\mathcal{M}}=\bigcup_{\theta \in \hat{\Gamma}} \operatorname{spec} \Delta_{M}^{\theta}=\bigcup_{k \in \mathbb{N}} B_{k}(\mathcal{M})
$$

where $B_{k}=B_{k}(\mathcal{M})=\left\{\lambda_{k}^{\theta}(M) ; \theta \in \hat{\Gamma}\right\}$ is a compact interval, called the $k$-th band (see e.g. [RS4, [Do1]) or [BrS]. Note that we have the following DirichletNeumann enclosure

$$
\lambda_{k}^{\mathrm{N}}(M) \leq \lambda_{k}^{\theta}(M) \leq \lambda_{k}^{\mathrm{D}}(M)
$$

which follows easily from the opposite inclusions of the corresponding quadratic form domains via the Min-max principle. Therefore, the $k$-th Dirichlet resp. Neumann eigenvalue are an upper resp. lower bound for the $k$-th band. In particular, if the interval $I_{k}$ defined in (1.1) is non-empty, it lies in a spectral gap of $\Delta_{\mathcal{M}}$. In general, we do not know whether the intervals $I_{k}$ are empty or not. In many cases, the Dirichlet-Neumann enclosure is too rough to guarantee the gap condition (1.1). Nevertheless, in Section 5 we cite examples where the gap condition holds.

\section{Elliptic estimates AND Perturbations of the Metric}

First, we construct an atlas of $\mathcal{M}$ adapted to the periodic structure. This enables us to define a "natural" norm on the Sobolev space on the non-compact periodic manifold $\mathcal{M}$. A more general concept requires only bounds on the Ricci curvature instead of the periodicity (cf. e.g. [He]). Next, we need some elliptic estimates. These estimates are used to obtain estimates in which different Laplacians (defined with respect to different metrics) occur.

Periodic atlases and Sobolev spaces. Suppose that $\left(\widetilde{U}_{\beta}\right), \beta \in B$, is a finite cover of $\mathcal{M} / \Gamma$ and that $\widetilde{\varphi}_{\beta}: \widetilde{U}_{\beta} \longrightarrow \widetilde{V}_{\beta}$ are charts with open sets $\widetilde{V}_{\beta} \subset \mathbb{R}^{d}$. We assume that all transition maps $\widetilde{\varphi}_{\beta_{1}} \circ \widetilde{\varphi}_{\beta_{2}}^{-1}$ (when defined) have bounded derivatives up to all orders. We can lift this atlas of the quotient to an atlas $\varphi_{\alpha}: U_{\alpha} \longrightarrow V_{\alpha}$, $\alpha:=(\beta, \gamma) \in A:=B \times \Gamma$ of the periodic manifold $\mathcal{M}$, i.e., $\widetilde{\varphi}_{\beta} \circ \pi=\varphi_{\alpha}$, $U_{(\beta, \gamma)}=\gamma U_{(\beta, 1)}$ and $\widetilde{V}_{\beta}=V_{\alpha}$. We call such an atlas $A$ periodic.

If we consider $\Gamma^{\prime} M$ instead of the full periodic manifold $\Gamma M=\mathcal{M}$ we set $V_{\alpha}^{\prime}:=\varphi_{\alpha}\left(U_{\alpha} \cap \Gamma^{\prime} M\right)$ which is an open set in $\mathbb{R}_{+}^{d}:=[0, \infty) \times \mathbb{R}^{d-1}$. Note that if $\Gamma^{\prime} \neq \Gamma$ then $\Gamma^{\prime} M$ has non-empty boundary being isometric to (copies of) $\partial M$. We also call the atlas $A^{\prime}:=B \times \Gamma^{\prime}$ of $\Gamma^{\prime} M$ with charts $\varphi_{\alpha}: U_{\alpha} \cap \Gamma^{\prime} M \longrightarrow V_{\alpha}^{\prime}$ periodic.

If $\widetilde{g}_{\beta}$ denotes the metric of $\mathcal{M} / \Gamma$ carried via the chart $\widetilde{\varphi}_{\beta}$ on $\widetilde{V}_{\beta}$ and $g_{\alpha}$ the periodic metric $g$ of $\mathcal{M}$ carried via $\varphi_{\alpha}$ on $V_{\alpha}=\widetilde{V}_{\beta}$ then $\widetilde{g}_{\beta}=g_{\alpha}$ for all $\gamma \in \Gamma$, i.e., the set of different metrics $\left\{g_{\alpha} \mid a \in A\right\}$ has only $|B|<\infty$ many elements although $A$ is not finite.

In the same way we can lift a partition of unity $\left(\widetilde{\chi}_{\beta}\right)$ subordinated to the cover $\left(\widetilde{U}_{\beta}\right)$ to a partition of unity $\left(\varphi_{\alpha}\right)$ subordinated to $\left(U_{\alpha}\right)$, i.e., we have $\widetilde{\chi}_{\beta} \circ \pi=\chi_{\alpha}$. 
We call such a partition of unity periodic. Again, the set of different functions $\left\{\chi_{\alpha} \circ \varphi_{\alpha}^{-1} \mid a \in A\right\}$ is finite.

The next definitions are useful for the elliptic estimate in Theorem 3.5.

Definition 3.1. A metric $h$ on $\Gamma^{\prime} M$ (not necessarily periodic) will be called uniformly elliptic (with respect to the atlas $A^{\prime}$ ) if there exists a constant $c>0$ such that

$$
c^{-1}|v|^{2} \leq h_{\alpha}(x)(v, v) \leq c|v|^{2}
$$

for all $v \in \mathbb{R}^{d}$, all $x \in V_{\alpha}^{\prime}$ and all $\alpha \in A^{\prime}$. We will also write $c^{-1} \leq h \leq c$ for short.

Next, we define a norm on the space of $C^{1}$-sections in the bundle of symmetric bilinear sections on $\Gamma^{\prime} M$. A Riemannian metric is an element of this space.

Definition 3.2. A metric $h$ will be called $C^{1}$-bounded on $\Gamma^{\prime} M$ (with respect to the atlas $\left.A^{\prime}\right)$ if

$$
\|h\|_{C^{1}}:=\sup _{\alpha}\left\|h_{\alpha}\right\|_{C^{1}}:=\sup _{\alpha} \sup _{x \in V_{\alpha}^{\prime}} \max _{i, j, k}\left\{\left|h_{\alpha, i j}(x)\right|,\left|\partial_{k} h_{\alpha, i j}(x)\right|\right\}
$$

is finite.

We let $\mathcal{G}\left(c, c_{1}, \Gamma^{\prime}\right)$ be the space of all uniformly elliptic and $C^{1}$-bounded metrics $h$ on $\Gamma^{\prime} M$ such that $c^{-1} \leq h \leq c$ and $\|h\|_{C^{1}} \leq c_{1}$.

Remark 3.3. Note that the atlas can always be chosen in such a way that a periodic metric $g$ on $\mathcal{M}$ is uniformly elliptic and $C^{1}$-bounded. If necessary we slightly need to make the charts smaller such that $g_{\alpha}$ has derivatives continuous up to the boundary of $V_{\alpha}$. In the same way, any metric $h$ on $\Gamma^{\prime} M$ is uniformly elliptic if $\Gamma^{\prime}$ is finite.

Remark 3.4. It seems to be unsatisfactory that our definitions depend on the atlas $A^{\prime}$. We also could define the uniform ellipticity in a coordinate free manner by comparing a metric $h$ with the periodic metric $g$ globally. Likewise, we could define the $C^{1}$-boundedness in a global way by defining how to derivative a metric. But since we have chosen a periodic atlas one can easily see that the local and global definitions are equivalent. For a similar concept of $C^{1}$-convergence of manifolds see e.g. [H]].

For $k \in \mathbb{N}$ and an open set $V^{\prime} \subset \mathbb{R}_{+}^{d}$ let $\mathcal{H}^{k}\left(V^{\prime}\right)$ be the usual Sobolev space of order $k$ defined as the closure of $C^{\infty}\left(V^{\prime}\right)$ under the norm

$$
\|u\|_{k, V^{\prime}}^{2}:=\sum_{|\kappa| \leq k}\left\|\partial_{\kappa} u\right\|_{V^{\prime}}^{2} .
$$

We define the Sobolev space $\mathcal{H}^{k}\left(\Gamma^{\prime} M\right)$ on $\Gamma^{\prime} M$ as the closure of the space of all functions $u \in C^{\infty}\left(\Gamma^{\prime} M\right)$ such that

$$
\|u\|_{k, \Gamma^{\prime} M}^{2}:=\sum_{\alpha \in A^{\prime}}\left\|u_{\alpha}\right\|_{k, V_{\alpha}^{\prime}}^{2}
$$


is finite. Here, $u_{\alpha}:=\left(\chi_{\alpha} u\right) \circ \varphi_{\alpha}^{-1}$ denotes the local representation of $\chi_{\alpha} u$ in the chart $\varphi_{\alpha}$. We denote by $\mathcal{H}^{k}\left(\Gamma^{\prime} M\right)$ the completion of $C_{\mathrm{c}}^{\infty}\left(\Gamma^{\prime} M\right)$, i.e., the space of smooth functions with compact support away from $\partial \Gamma^{\prime} M$, under the norm defined in (3.3).

Since the periodic metric $g$ is uniformly elliptic, we conclude

$$
c^{-d / 4}\|u\|_{\Gamma^{\prime} M} \leq\|u\|_{0, \Gamma^{\prime} M}=\sum_{\alpha}\left\|u_{\alpha}\right\|_{V_{\alpha}} \leq c^{+d / 4}|B|(2 r+1)\|u\|_{\Gamma^{\prime} M},
$$

i.e., $\|\cdot\|_{\Gamma^{\prime} M}$ and $\|\cdot\|_{0, \Gamma^{\prime} M}$ are equivalent norms. Remember that $r$ denotes the number of generators of $\Gamma$. Note that a chart $U_{\alpha}$ could intersect with at most $|B|(2 r+1)$ other charts. In the same way we can show that $\left(\|u\|^{2}+\|\mathrm{d} u\|^{2}\right)^{\frac{1}{2}}$ and $\|u\|_{1, \Gamma^{\prime} M}$ are equivalent norms on $\mathcal{H}^{1}\left(\Gamma^{\prime} M\right)$. In particular, we have $\mathcal{H}^{1}\left(\Gamma^{\prime} M\right)=$ $\operatorname{dom} q_{\Gamma^{\prime} M}^{\mathrm{D}}$ and $\mathcal{H}^{1}\left(\Gamma^{\prime} M\right)=\operatorname{dom} q_{\Gamma^{\prime} M}^{\mathrm{N}}$.

Elliptic estimates. The main result of this subsection is the following theorem:

Theorem 3.5. For every $c>0, c_{1}>0$ and $d>0$ there exists a constant $c_{2}$ such that

$$
\|u\|_{2, U} \leq c_{2}\left(\|u\|_{\Gamma^{\prime} M}+\left\|\Delta^{\prime} u\right\|_{\Gamma^{\prime} M}\right)
$$

for all $u \in \mathcal{H}^{2}(U)$, all metrics $g^{\prime} \in \mathcal{G}\left(c, c_{1}, \Gamma^{\prime}\right)$, all open sets $U \subset \Gamma^{\prime} M$ such that $\operatorname{dist}\left(U, \partial \Gamma^{\prime} M\right) \geq d$ and all $\Gamma^{\prime} \subset \Gamma$. Here, $\Delta^{\prime}$ denotes the Laplacian on $\Gamma^{\prime} M$ with respect to the metric $g^{\prime}$.

If $\partial M$ is smooth Estimate (3.5) is valid for $U=\Gamma^{\prime} M$, all $u \in \mathcal{H}^{2}\left(\Gamma^{\prime} M\right) \cap$ $\mathcal{H}^{1}\left(\Gamma^{\prime} M\right)$, all metrics $g^{\prime} \in \mathcal{G}\left(c, c_{1}, \Gamma^{\prime}\right)$ and all $\Gamma^{\prime} \subset \Gamma$.

Proof. The proof of the statement in the chart $V_{\alpha}^{\prime}$ is standard (see e.g. [GiT]). The step from the local to the global estimate is possible since the constant in the local estimate has a global bound. Here, we need Estimate (3.4) and therefore the special structure of the atlas and the partition of unity $\left(\chi_{\alpha}\right)$. Furthermore, we have to estimate the first order operators $\left[\Delta^{\prime}, \chi_{\alpha}\right]$ in terms of the right hand side of (3.5). This can be done applying the Gauß-Green formula. Note that $u$ vanishes on the boundary.

The proof of the next lemma is straightforward. Again, we need (3.4) for the step from the local to the global estimate.

Lemma 3.6. There exists a constant $c_{3}>0$ such that

$$
\left\|\Delta_{M^{n}} u\right\|_{M^{m}} \leq c_{3}\|u\|_{2, M^{m}}
$$

for all $u \in \mathcal{H}^{2}\left(M^{n}\right)$ and all $m, n$ with $n>m$. Suppose further that $g_{\tau}, \tau \geq 0$, are metrics on $\mathcal{M}$ such that $\left\|g_{0}-g_{\tau}\right\|_{C^{1}} \rightarrow 0$ on $R^{m, n}$ as $m \rightarrow \infty(n>m)$ uniformly in $\tau \geq 0$. Then there exists a sequence $\delta_{m}^{\prime} \rightarrow 0$ such that

$$
\left\|\left(\Delta_{M_{0}^{n}}-\Delta_{M_{\tau}^{n}}\right) u\right\|_{R^{m, n}} \leq \delta_{m}^{\prime}\|u\|_{2, R^{m, n}}
$$

for all $u \in \mathcal{H}^{2}\left(R^{m, n}\right)$, all $\tau \geq 0$ and all $m, n$ with $n>m$. 
Let $m_{+}$be the smallest integer such that $M^{m} \subset \stackrel{\circ}{M}^{m_{+}}$. Now we prove the estimates needed in the next section:

Lemma 3.7. Let the family $\left(g_{\tau}\right)$ satisfy Conditions (1.2) and (1.3). Suppose further that $\tau_{n} \rightarrow \tau_{0} \geq 0$. Then there exists a constant $c_{4}>0$ such that

$$
\left\|\Delta_{M^{n}} u\right\|_{M^{m}} \leq c_{4}\left(\|u\|_{M^{m_{+}}}+\left\|\Delta_{M_{\tau_{n}}^{n}} u\right\|_{M^{m_{+}}}\right)
$$

for all $u \in \mathcal{H}^{2}\left(M^{n}\right)$ and all $m, n$ with $n>m_{+}$. Furthermore if in addition $\partial M$ is smooth and if Condition (1.4) is satisfied then there exists a sequence $\delta_{m} \rightarrow 0$ such that

$$
\left\|\left(\Delta_{M^{n}}-\Delta_{M_{\tau_{n}}^{n}}\right) u\right\|_{R^{m, n}} \leq \delta_{m}\left(\|u\|_{R^{m, n}}+\left\|\Delta_{M_{\tau_{n}}^{n}} u\right\|_{R^{m, n}}\right)
$$

for all $u \in \mathcal{H}^{2}\left(R^{m, n}\right) \cap \mathcal{H}^{1}\left(R^{m, n}\right)$ and all $m, n$ with $n>m$ and $m$ large enough.

Proof. For the proof of (3.6) we combine Lemma 3.6 with Theorem 3.5. Here we need a cut-off function $\chi \in C_{\mathrm{c}}^{\infty}\left(M^{m_{+}}\right)$such that $\chi \uparrow M^{m}=1$. Therefore $\operatorname{dist}\left(\operatorname{supp} \chi, \partial M^{m}\right)=d>0$, i.e., we do not need the assumption that $\partial M$ is smooth in this case. By Remark 3.3, there exist $c, c_{1}>0$ such that $g_{\tau_{0}} \in \mathcal{G}\left(c, c_{1}, \Gamma^{m_{+}}\right)$. Furthermore, Condition (1.3) assures that there exist constants $c^{\prime}, c_{1}^{\prime}>0$ such that $g_{\tau_{n}} \in \mathcal{G}\left(c^{\prime}, c_{1}^{\prime}, \Gamma^{m_{+}}\right)$for all $n \in \mathbb{N}$. Note that we still have to estimate the first order operator $\left[\chi, \Delta_{M_{\tau_{n}}^{n}}\right]$ in term of the right hand side of (3.6) as in the proof of Theorem 3.5. Here we also need the fact that $g_{\tau_{n}} \in \mathcal{G}\left(c^{\prime}, c_{1}^{\prime}, \Gamma^{m_{+}}\right)$.

The proof of (3.7) is similar. Note that $g_{\tau_{n}}, n \in \mathbb{N}$, are close to the periodic metric $g=g_{0}$ by Condition (1.4) on $R^{m, n}$ provided $m$ is large enough. Since the periodic metric $g$ is uniformly elliptic, there exist constants $c, c_{1}>0$ such that $g_{\tau_{n}} \in \mathcal{G}\left(c, c_{1}, \Gamma^{n} \backslash \Gamma^{m}\right)$ for all $n>m$.

Perturbation of the metric. Here, we state some results on how to deal with the different Hilbert spaces $L_{2}\left(\mathcal{M}_{\tau}\right)$ resp. $\mathcal{H}^{1}\left(\mathcal{M}_{\tau}\right)$ depending on $\tau$. In particular, we show that the norm and weak topology on $L_{2}\left(\mathcal{M}_{\tau}\right)$ resp. $\mathcal{H}^{1}\left(\mathcal{M}_{\tau}\right)$ are equivalent for all $\tau \geq 0$; the same is true on the submanifolds $\Gamma^{\prime} M$ (cf. Corollary 3.9). Furthermore, we need the continuity of the eigenvalues with respect to $\tau$, see Corollary 3.10 and Lemma 3.11 .

Lemma 3.8. Suppose that the family $\left(g_{\tau}\right)$ satisfies Condition (1.3). Suppose furthermore that $\tau_{n} \rightarrow \tau \geq 0$. Then there exists a sequence $\eta_{n} \rightarrow 0$ such that

$$
\begin{aligned}
\left|\|u\|_{\mathcal{M}_{\tau}}^{2}-\|u\|_{\mathcal{M}_{\tau_{n}}}^{2}\right| & \leq \eta_{n}\|u\|_{\mathcal{M}_{\tau}}^{2} \\
\left|\|\mathrm{~d} u\|_{\mathcal{M}_{\tau}}^{2}-\|\mathrm{d} u\|_{\mathcal{M}_{\tau_{n}}}^{2}\right| & \leq \eta_{n}\|\mathrm{~d} u\|_{\mathcal{M}_{\tau}}^{2}
\end{aligned}
$$

for all $u \in L_{2}(\mathcal{M})$ resp. $u \in \mathcal{H}^{1}(\mathcal{M})$. 
Proof. We only give the idea of how to prove (3.9). The other inequality can be proven similarly. We have

$$
\begin{aligned}
& \int_{\mathcal{M}_{\tau}}|\mathrm{d} u|^{2}-\int_{\mathcal{M}_{\tau_{n}}}|\mathrm{~d} u|^{2}= \\
& \quad=\sum_{\alpha} \int_{V_{\alpha}} \chi_{\alpha}\left(\left(E-\left(\frac{\operatorname{det} G_{\alpha, n}}{\operatorname{det} G_{\alpha}}\right)^{\frac{1}{2}} G_{\alpha}^{\frac{1}{2}} G_{\alpha, n}^{-1} G_{\alpha}^{\frac{1}{2}}\right) G_{\alpha}^{-\frac{1}{2}} \nabla u \cdot G_{\alpha}^{-\frac{1}{2}} \nabla u\right)\left(\operatorname{det} G_{\alpha}\right)^{\frac{1}{2}} .
\end{aligned}
$$

Here, $G_{\alpha}$ resp. $G_{\alpha, n}$ denotes the matrix corresponding to $g_{\tau}$ resp. $g_{\tau_{n}}$ in the chart indexed by $\alpha$. Furthermore, $E$ denotes the unit $(d \times d)$-matrix. Clearly, Estimate (3.9) follows from Condition (1.3).

The next corollary follows easily.

Corollary 3.9. For all $\tau_{0} \geq 0$ there exists a constants $c_{5}=c_{5}\left(\tau_{0}\right) \geq 1$ such that

$$
\begin{gathered}
c_{5}^{-1}\|u\|_{\mathcal{M}_{0}}^{2} \leq\|u\|_{\mathcal{M}_{\tau}}^{2} \leq c_{5}\|u\|_{\mathcal{M}_{0}}^{2} \\
c_{5}^{-1}\|\mathrm{~d} u\|_{\mathcal{M}_{0}}^{2} \leq\|\mathrm{d} u\|_{\mathcal{M}_{\tau}}^{2} \leq c_{5}\|\mathrm{~d} u\|_{\mathcal{M}_{0}}^{2}
\end{gathered}
$$

for all $0 \leq \tau \leq \tau_{0}$.

From Lemma 3.8 and the Min-max principle we conclude the following corollary.

Corollary 3.10. The eigenvalue branch $\tau \mapsto \lambda_{k}^{\mathrm{D}}\left(M_{\tau}^{n}\right)$ is continuous for $\tau \geq 0$. In particular, for every $\tau_{0} \geq 0$ there exist a modul of continuity for the eigenvalue branch on $\left[0, \tau_{0}\right]$, i.e. a non-negative monotonical increasing function $\eta$ with $\eta(\delta) \rightarrow 0$ as $\delta \rightarrow 0$ such that

$$
\left|\lambda_{k}^{\mathrm{D}}\left(M_{\tau}^{n}\right)-\lambda_{k}^{\mathrm{D}}\left(M_{\tau^{\prime}}^{n}\right)\right| \leq \eta\left(\left|\tau-\tau^{\prime}\right|\right)
$$

for all $\tau, \tau^{\prime} \in\left[0, \tau_{0}\right]$.

In the same way we can show that the boundary conditions on $R_{\tau}^{m, n}$ have nearly no influence:

Lemma 3.11. Suppose that the family $\left(g_{\tau}\right)$ of metrics satisfies Condition (1.4]). Then there exists a sequence $\eta_{m} \rightarrow 0$ as $m \rightarrow \infty$ such that

$$
\left|\lambda_{k}^{\mathrm{D}}\left(R_{\tau}^{m, n}\right)-\lambda_{k}^{\mathrm{D}}\left(R^{m, n}\right)\right| \leq \eta_{m}
$$

for all $\tau \geq 0$ and $n>m$. The same estimate is true for Neumann or mixed boundary conditions on $R^{m, n}$.

\section{Eigenvalues in Gaps}

In this section we prove our main result Theorem 1.1. We approximate eigenfunctions of the perturbed Laplacian on the full manifold $\mathcal{M}$ by eigenfunctions of the perturbed Dirichlet-Laplacian on the approximating manifold $M^{n}$. This idea has already been used in [DH], [ADH] or [AADH]. Our situation is in some sense 
simpler: the approximating and the limit problem have the same spectral gap. Otherwise our situation is more difficult since not only the Laplacian but also the Hilbert space depend on the perturbation parameter. Some of the technical details are already proven in Section 3 .

In Theorem 4.3 we show the convergence of the approximating eigenfunctions. Furthermore, in Theorem 4.5 we give a lower bound on the eigenvalue counting function defined in (11.5) following arguments of [HB].

Firstly, we use the decomposition principle (see [DoL]) to prove that the essential spectrum remains invariant under the perturbation. Therefore, $\Delta_{\mathcal{M}}$ and $\Delta_{\mathcal{M}_{\tau}}$ have the same spectral gap. In a spectral gap of the unperturbed Laplacian, the perturbed Laplacian can only have discrete eigenvalues (possibly accumulating at the band edges). It is essential here that the perturbation is (almost) localized on a compact set.

Theorem 4.1. We have ess spec $\Delta_{\mathcal{M}}=$ ess spec $\Delta_{\mathcal{M}_{\tau}}$ for all $\tau \geq 0$.

Proof. We only show the inclusion " $\subset$ " since the opposite inclusion can be proven in the same way.

Let $\left(u_{n}\right)$ be a singular sequence for $\lambda \in \operatorname{ess} \operatorname{spec} \Delta_{\mathcal{M}}$, i.e., $u_{n} \rightarrow 0$ weakly in $L_{2}(\mathcal{M}),\left\|u_{n}\right\|_{\mathcal{M}}=1$ and $\left\|\left(\Delta_{\mathcal{M}}-\lambda\right) u_{n}\right\|_{\mathcal{M}} \rightarrow 0$ as $n \rightarrow \infty$. By the decomposition principle (cf. DoD] or [P1]) we can assume that $u_{n}$ has support away from $M^{m}$. Furthermore, by Corollary 3.9 there exists $c_{5}>0$ such that $\left\|u_{n}\right\|_{\mathcal{M}_{\tau}} \geq c_{5}^{-1}$ for all $n$.

Now we want to show that $v_{n}:=u_{n} /\left\|u_{n}\right\|_{\mathcal{M}_{\tau}}$ is a singular sequence for $\lambda$ and $\Delta_{\mathcal{M}_{\tau}}$. Clearly, $v_{n} \rightarrow 0$ weakly in $L_{2}\left(\mathcal{M}_{\tau}\right)$. Since $u_{n} \uparrow M^{m}=0$ we have

$$
\begin{aligned}
\left\|\left(\Delta_{\mathcal{M}_{\tau}}-\lambda\right) v_{n}\right\|_{\mathcal{M}_{\tau}} & \leq c_{5}\left(\left\|\left(\Delta_{\mathcal{M}_{\tau}}-\Delta_{\mathcal{M}}\right) u_{n}\right\|_{R^{m}}+\left\|\left(\Delta_{\mathcal{M}}-\lambda\right) u_{n}\right\|_{R^{m}}\right) \\
& \leq \delta_{m} c_{5}\left(\left\|u_{n}\right\|_{R^{m}}+\left\|\Delta_{\mathcal{M}} u_{n}\right\|_{R^{m}}\right)+c_{5}\left\|\left(\Delta_{\mathcal{M}}-\lambda\right) u_{n}\right\|_{R^{m}}
\end{aligned}
$$

by an estimate similar to (3.7). Here, $\delta_{m} \rightarrow 0$ as $m \rightarrow \infty$. Note that we do not need the smoothness of $\partial M$ here even if the perturbation lives on the whole manifold since supp $u_{n}$ is compactly contained in $R^{m}$. Therefore the first term converges to 0 . The last term converges to 0 since $\left(u_{n}\right)$ is a singular sequence for $\Delta_{\mathcal{M}}$

Next, we conclude from the gap condition (1.1) and the Dirichlet-Neumann enclosure (2.4) that no eigenvalue of the approximating problem lies in the gap. The boundary of $M$ resp. $\Gamma^{\prime} M$ is so small such that boundary conditions almost have no influence on the eigenvalues.

Lemma 4.2. Suppose that $\Gamma^{\prime}$ has $n$ elements. If $\lambda_{k}^{\mathrm{D}}(M)<\lambda_{k+1}^{\mathrm{N}}(M)$ then the interval $I_{k}=\left(\lambda_{k}^{\mathrm{D}}(M), \lambda_{k+1}^{\mathrm{N}}(M)\right)$ is a common spectral gap, i.e.,

$$
I_{k} \cap \operatorname{spec} \Delta_{\mathcal{M}}=\emptyset \quad \text { and } \quad I_{k} \cap \operatorname{spec} \Delta_{\Gamma^{\prime} M}=\emptyset .
$$


Here, $\Delta_{\Gamma^{\prime} M}$ denotes the Laplacian on $\Gamma^{\prime} M$ with Neumann, Dirichlet or mixed boundary condition on $\partial \Gamma^{\prime} M$. Furthermore, if $\lambda \in I_{k}$ then $\operatorname{dim}_{\lambda}\left(\Gamma^{\prime} M\right)=k n$ (also for any boundary condition).

Proof. Denote by $\Sigma^{n} M$ the disjoint union of $n$ copies of $M$. Then $\lambda_{j}^{\mathrm{D}}\left(\Sigma^{n} M\right)=$ $\lambda_{k}^{\mathrm{D}}(M)$ and $\lambda_{j}^{\mathrm{N}}\left(\Sigma^{n} M\right)=\lambda_{k}^{\mathrm{N}}(M)$ for all $j=(k-1) n+1, \ldots, k n$ since every eigenvalue on the disjoint union has multiplicity $p n$ if the corresponding eigenvalue on $M$ has multiplicity $p$. By the Dirichlet-Neumann bracketing (2.3) we obtain

$$
\lambda_{k}^{\mathrm{N}}(M)=\lambda_{j}^{\mathrm{N}}\left(\Sigma^{n} M\right) \leq \lambda_{j}\left(\Gamma^{\prime} M\right) \leq \lambda_{j}^{\mathrm{D}}\left(\Sigma^{n} M\right)=\lambda_{k}^{\mathrm{D}}(M) .
$$

Note that this estimate is true for any boundary condition on $\Gamma^{\prime} M$. Together with the Dirichlet-Neumann enclosure (2.4) we see that $\lambda_{k}^{\mathrm{N}}(M)$ resp. $\lambda_{k}^{\mathrm{D}}(M)$ are lower resp. upper bounds for both $\lambda_{j}\left(\Gamma^{\prime} M\right)$ and $\lambda_{k}^{\theta}(M)$. If $\lambda \in I_{k}$ then $\Delta_{\Gamma^{\prime} M}$ has exactly $k n$ eigenvalues below $\lambda$.

Now we prove that approximating eigenfunctions converge to eigenfunctions of the full problem and that multiplicity is conserved as $n \rightarrow \infty$, i.e., as the number of copies of period cells increases. Here and later on we suppress the Dirichlet label at the approximating problem, e.g. we write $\Delta_{M^{n}}$ instead of $\Delta_{M^{n}}^{\mathrm{D}}$ until stated otherwise.

The idea of the next theorem is from [DH, [ADH] or AADH]:

Theorem 4.3. Suppose that the gap condition (1.1) is satisfied, i.e., $I_{k}$ is a spectral gap of the periodic Laplacian $\Delta_{\mathcal{M}}$ for some $k \in \mathbb{N}$. Suppose further that the family $\left(g_{\tau}\right)$ satisfies Conditions (1.2), (1.3) and (1.4) or, if $\partial M$ is smooth, (1.4]). Let $\tau_{n} \rightarrow \tau, \lambda_{n, i} \rightarrow \lambda \in I_{k}$ for all $i=1, \ldots, j$. Furthermore, suppose that $\varphi_{n, 1}, \ldots, \varphi_{n, j} \in L_{2}\left(M_{\tau_{n}}^{n}\right)$ are orthonormal for all $n \in \mathbb{N}$ and that

$$
\Delta_{M_{\tau_{n}}^{n}} \varphi_{n, i}=\lambda_{n, i} \varphi_{n, i} .
$$

Then for every $i$ there exists a subsequence of $\left(\varphi_{n, i}\right)_{n}$ also denoted by $\left(\varphi_{n, i}\right)_{n}$ converging weakly to $\varphi_{0, i} \in \operatorname{dom} \Delta_{\mathcal{M}_{\tau}}$ in $\mathcal{H}^{1}\left(\mathcal{M}_{\tau}\right)$ and strongly in $L_{2, \operatorname{loc}}\left(\mathcal{M}_{\tau}\right)$. Furthermore,

$$
\Delta_{\mathcal{M}_{\tau}} \varphi_{0, i}=\lambda \varphi_{0, i}
$$

and $\varphi_{0,1}, \ldots, \chi_{0, j}$ are linearly independent.

Proof. Let $i \in\{1, \ldots j\}$. From Corollary 3.9 it follows that $\left(\varphi_{n, i}\right)_{n}$ is bounded in $\mathcal{H}^{1}(\mathcal{M})$. Therefore we can extract a subsequence also denoted by $\left(\varphi_{n, i}\right)_{n}$ converging weakly in $\mathcal{H}^{1}(\mathcal{M})$ to an element $\varphi_{0, i}$. This subsequence also converges strongly in $L_{2, \text { loc }}(\mathcal{M})$ by the Rellich-Kondrachov compactness Theorem. In virtue of Lemma 3.8 and Corollary 3.9 it is a straightforward calculation to show that $\varphi_{0, i}$ is in the domain of $\Delta_{\mathcal{M}_{\tau}}$ and that the eigenvalue equation (4.3) is satisfied.

The main difficulty is to prove that $\varphi_{0, i}$ are linearly independent. Suppose that there exist $\alpha_{1}, \ldots, \alpha_{k}$, not all equal to 0 , such that $u_{n}:=\sum_{i} \alpha_{i} \varphi_{n, i}$ converges to $\sum_{i} \alpha_{i} \varphi_{0, i}=0$ in $L_{2, \text { loc }}\left(\mathcal{M}_{\tau}\right)$. By Lemma 4.2 the interval $I_{k}$ is a spectral gap for 
all operators $\Delta_{M^{n}}, n \in \mathbb{N}$. In virtue of the spectral theorem and Estimate (3.10) we have

$$
\left\|\left(\Delta_{M^{n}}-\lambda\right) u_{n}\right\|_{M^{n}}^{2} \geq c_{6}^{2}\left\|u_{n}\right\|_{M^{n}}^{2} \geq \frac{c_{6}^{2}}{c_{5}} \sum_{i}\left|\alpha_{i}\right|^{2}>0
$$

for all $n \in \mathbb{N}$ where $c_{6}:=\operatorname{dist}\left(\lambda, \mathbb{R} \backslash I_{k}\right)$. On the other side we have

$\left\|\left(\Delta_{M^{n}}-\lambda\right) u_{n}\right\|_{M^{n}} \leq\left\|\sum_{i}\left(\Delta_{M^{n}}-\lambda_{n, i}\right) \alpha_{i} \varphi_{n, i}\right\|_{M^{n}}+\sum_{i}\left|\alpha_{i}\right| \cdot\left|\lambda_{n, i}-\lambda\right| \cdot\left\|\varphi_{n, i}\right\|_{M^{n}}$.

The last sum converges to 0 since $\left\|\varphi_{n, i}\right\|_{M^{n}} \leq c_{5}$. The first norm can be split into an integral over $M^{m}$ and $R^{m, n}$.

The integral over $M^{m}$ can be estimated by

$$
\left\|\Delta_{M^{n}} u_{n}\right\|_{M^{m}}+\sum_{i} \lambda_{n, i}\left\|u_{n}\right\|_{M^{m}}
$$

Furthermore, Inequality (3.6) yields the estimate

$$
\begin{aligned}
\left\|\Delta_{M^{n}} u_{n}\right\|_{M^{m}} \leq c_{4} c_{5}\left(\left\|u_{n}\right\|_{M_{\tau}^{m_{+}}}+\left\|\Delta_{M_{\tau_{n}}^{n}} u_{n}\right\|_{M_{\tau}^{m_{+}}}\right) & \\
& \leq c_{4} c_{5}\left(\left(1+\sum_{i} \lambda_{n, i}\right)\right)\left\|u_{n}\right\|_{M_{\tau}^{m_{+}}}
\end{aligned}
$$

for all $n>m_{+}$. In the last estimate we have used Equation (4.2). Note that the last term converges to 0 since $u_{n} \rightarrow 0$ in $L_{2, \text { loc }}(\mathcal{M})$.

If the perturbation is contained in $M^{m}$ we have a contradiction to (4.4). Otherwise we still have to estimate the integral over $R^{m, n}$. Here, we need the smoothness assumption on $\partial M$ to be able to use (3.7). Together with (4.2) we obtain

$$
\begin{aligned}
\left\|\left(\Delta_{M^{n}}-\Delta_{M_{\tau_{n}}^{n}}\right) u_{n}\right\|_{R^{m, n}} \leq \delta_{m}\left(\left\|u_{n}\right\|_{M^{n}}+\right. & \left.\left\|\Delta_{M_{\tau_{n}}^{n}} u_{n}\right\|_{M^{n}}\right) \\
& \leq \delta_{m} c_{5}\left(\sum_{i}\left|\alpha_{i}\right|^{2}\right)^{\frac{1}{2}}\left(1+\max _{i} \lambda_{n, i}\right)
\end{aligned}
$$

tending to 0 as $m \rightarrow \infty$. Again, we have a contradiction to (4.4).

The next result allows us to estimate the eigenvalue counting functions for different parameters $\tau$ and fixed $\lambda$ by the eigenvalue counting function for a fixed $\tau$ and an interval containing $\lambda$ (see $[\mathrm{HB}$ ). We need this lemma since we do not know whether eigenfunctions corresponding to different parameters $\tau$ are orthogonal. We do not even know that they are different! But since we want to show that the multiplicity of eigenvalues is conserved as $n \rightarrow \infty$ we need the linear independence of the approximating eigenfunctions (see the proof of Theorem 4.5).

Lemma 4.4. For all $\tau_{0} \geq 0$ there exists a monotonical increasing function $\eta(\delta) \rightarrow 0$ as $\delta \rightarrow 0$ such that

$$
\left|\operatorname{dim}_{\lambda}^{\mathrm{D}}\left(M_{\tau+\delta}^{n}\right)-\operatorname{dim}_{\lambda}^{\mathrm{D}}\left(M_{\tau-\delta}^{n}\right)\right| \leq \operatorname{dim}_{[\lambda-\eta(\delta), \lambda+\eta(\delta)]}^{\mathrm{D}}\left(M_{\tau}^{n}\right)
$$


for all $\lambda \geq 0, \delta>0$ and all $\tau+\delta \leq \tau_{0}$.

Proof. Since every eigenvalue branch $\lambda_{j}^{\mathrm{D}}\left(M_{(\cdot)}^{n}\right)$ is continuous by Corollary 3.10, there exists a parameter $\tau^{\prime} \in[\tau-\delta, \tau+\delta]$ such that $\lambda=\lambda_{j}^{\mathrm{D}}\left(M_{\tau^{\prime}}^{n}\right)$ by the intermediate value theorem. Corollary 3.10 yields

$$
\left|\lambda-\lambda_{j}^{\mathrm{D}}\left(M_{\tau}^{n}\right)\right|=\left|\lambda_{j}^{\mathrm{D}}\left(M_{\tau^{\prime}}^{n}\right)-\lambda_{j}^{\mathrm{D}}\left(M_{\tau}^{n}\right)\right| \leq \eta\left(\left|\tau-\tau^{\prime}\right|\right) \leq \eta(\delta),
$$

i.e., $\Delta_{M_{\tau}^{n}}$ has an eigenvalue in $[\lambda-\eta(\delta), \lambda+\eta(\delta)]$.

Now we give a lower bound on the eigenvalue counting function following [HB]:

Theorem 4.5. If $\lambda \in I_{k}$ lies in a spectral gap then

$$
\mathcal{N}(\tau, \lambda) \geq \limsup _{n \rightarrow \infty}\left|\operatorname{dim}_{\lambda}^{\mathrm{D}}\left(M_{\tau}^{n}\right)-\operatorname{dim}_{\lambda}^{\mathrm{D}}\left(M^{n}\right)\right|
$$

for all $\tau \geq 0$.

Proof. Denote by

$$
T_{n}:=T_{n}(\lambda):=\left\{\tau^{\prime} \in\left[0, \tau_{0}\right] \mid \lambda \in \operatorname{spec} \Delta_{M_{\tau}^{n}}\right\},
$$

the set of parameters $\tau^{\prime}$ that produce an eigenvalue $\lambda$. Let $T_{\infty}$ be the set of limit points, i.e., $\hat{\tau} \in T_{\infty}$ if and only if $\hat{\tau} \in\left[0, \tau_{0}\right]$ and if there exist sequences $\left(n_{m}\right)_{m} \subset \mathbb{N}$ and $\tau_{m}^{\prime} \in T_{n_{m}}$ such that $\tau_{m}^{\prime} \rightarrow \hat{\tau}$.

We have to distinguish two cases. If the cardinality of $T_{\infty}$ is greater or equal to $N_{\lambda}$, the right hand side of (4.5), then we apply Theorem 4.3 with fixed eigenvalue $\lambda=\lambda_{n, 1}$ and with multiplicity $j=1$ for each limit point $\hat{\tau} \in T_{\infty}$. As a consequence, there are at least card $T_{\infty}$ parameters $\hat{\tau}$ such that $\lambda$ is an eigenvalue of $\Delta_{M(\hat{\tau})}$. This proves (4.5).

If card $T_{\infty}<N_{\lambda}$ then $T_{\infty}$ consists of a finite number of points $\hat{\tau}_{1}, \ldots, \hat{\tau}_{q}$, and $T_{n} \rightarrow\left\{\hat{\tau}_{1}, \ldots, \hat{\tau}_{q}\right\}$. Furthermore, there exists a sequence $\delta_{n} \rightarrow 0$ such that

$$
T_{n} \subset \bigcup_{p=1}^{q}\left(\hat{\tau}_{p}-\delta_{n}, \hat{\tau}_{p}+\delta_{n}\right)=: \hat{T}_{n}
$$

for all $n \in \mathbb{N}$. If $n$ is large enough, all these intervals are mutually disjoint. As a consequence, $d_{\lambda, n}(\hat{\tau}):=\operatorname{dim}_{\lambda}^{\mathrm{D}}\left(M_{\hat{\tau}}^{n}\right)$ is constant on each component of $[0, \tau] \backslash \hat{T}_{n}$. Therefore

$$
\left|d_{\lambda, n}(\tau)-d_{\lambda, n}(0)\right| \leq \sum_{p=1}^{q}\left|d_{\lambda, n}\left(\hat{\tau}_{p}-\delta_{n}\right)-d_{\lambda, n}\left(\hat{\tau}_{p}+\delta_{n}\right)\right|
$$

By Lemma 4.4 there exist $\eta(\delta) \rightarrow 0$ as $\delta \rightarrow 0$ such that

$$
\left|d_{\lambda, n}(\tau)-d_{\lambda, n}(0)\right| \leq \sum_{p=1}^{q} j_{p, n} \quad \text { where } \quad j_{p, n}:=\operatorname{dim}_{\left[\lambda-\eta\left(\delta_{n}\right), \lambda+\eta\left(\delta_{n}\right)\right]}^{\mathrm{D}}\left(M_{\tau_{p}}^{n}\right)
$$

By passing to a subsequence we can assume that $\left|d_{\lambda, n}(\tau)-d_{\lambda, n}(0)\right| \rightarrow N_{\lambda}$. We have even equality if $n$ is large enough since the sequence consists of integers. Now 
we select another subsequence $\left(n_{m}\right)_{m}$ such that $j_{p}:=j_{p, n_{m}} \in \mathbb{N}$ is independent of $m$. This is possible by choosing a convergent subsequence. Therefore, for each $m \in \mathbb{N}$, we have $j_{p}$ orthonormal eigenfunctions $\varphi_{m, 1}, \ldots, \varphi_{m, j_{p}}$ of the Laplacian on $M_{\hat{\tau}_{p}}^{n_{m}}$ with eigenvalues $\lambda_{m, i} \rightarrow \lambda$ as $m \rightarrow \infty$. Hence we can apply Theorem 4.3 with fixed parameter $\hat{\tau}_{p}$ and conclude that the limit problem $\Delta_{\mathcal{M}_{\tau}}$ has $\lambda$ as eigenvalue of multiplicity $j_{p}$. This proves the theorem since $\lambda$ hat multiplicity at least $\sum_{p=1}^{q} j_{p} \geq N_{\lambda}$.

Finally we want a lower bound on the eigenvalue counting function in terms of $M^{m}$ and not in terms of $M^{n}$

Lemma 4.6. For $m$ sufficiently large we have

$$
\begin{aligned}
& \operatorname{dim}_{\lambda}^{\mathrm{D}}\left(M_{\tau}^{n}\right)-\operatorname{dim}_{\lambda}^{\mathrm{D}}\left(M^{n}\right) \geq \operatorname{dim}_{\lambda}^{\mathrm{D}}\left(M_{\tau}^{m}\right)-\operatorname{dim}_{\lambda}^{\mathrm{D}}\left(M^{m}\right) \\
& \operatorname{dim}_{\lambda}^{\mathrm{D}}\left(M^{n}\right)-\operatorname{dim}_{\lambda}^{\mathrm{D}}\left(M_{\tau}^{n}\right) \geq \operatorname{dim}_{\lambda}^{\mathrm{N}}\left(M^{m}\right)-\operatorname{dim}_{\lambda}^{\mathrm{N}}\left(M_{\tau}^{m}\right)
\end{aligned}
$$

for all $\tau \geq 0$ and $n>m$.

Proof. From Lemma 4.2 we know that $\operatorname{dim}_{\lambda}\left(M^{n}\right)=k n$ and $\operatorname{dim}_{\lambda}\left(R^{m, n}\right)=k(n-$ $m)$ if $\lambda \in I_{k}$ independently of the boundary conditions. Furthermore, from Lemma 3.11 we obtain

$$
\operatorname{dim}_{\lambda}^{\mathrm{D}}\left(R_{\tau}^{m, n}\right)=\operatorname{dim}_{\lambda}^{\mathrm{D}}\left(R^{m, n}\right), \quad n>m, \tau \geq 0
$$

if $m$ is large enough. Furthermore, the Dirichlet-Neumann bracketing (2.3) yields

$$
\begin{aligned}
\operatorname{dim}_{\lambda}^{\mathrm{D}}\left(M_{\tau}^{n}\right)-\operatorname{dim}_{\lambda}^{\mathrm{D}}\left(M^{n}\right) & \\
& \geq \operatorname{dim}_{\lambda}^{\mathrm{D}}\left(M_{\tau}^{m}\right)+\operatorname{dim}_{\lambda}^{\mathrm{D}}\left(R_{\tau}^{m, n}\right)-\left(\operatorname{dim}_{\lambda}^{\mathrm{D}}\left(M^{m}\right)+\operatorname{dim}_{\lambda}^{\mathrm{ND}}\left(R^{m, n}\right)\right) \\
& =\operatorname{dim}_{\lambda}^{\mathrm{D}}\left(M_{\tau}^{m}\right)-k m .
\end{aligned}
$$

where $\operatorname{dim}_{\lambda}^{\mathrm{ND}}\left(R^{m, n}\right)$ denotes the eigenvalue counting function of the Laplacian with Dirichlet boundary condition on $\partial M^{n}$ and Neumann boundary condition on $\partial R^{m, n} \backslash \partial M^{n}$. Estimate (4.7) can be shown similarly.

To summarize: Theorem 4.1 ensures that a given spectral gap in the essential spectrum of the periodic manifold remains invariant under local perturbations. Therefore, $\mathcal{N}(\tau, \lambda)$ indeed counts the discrete eigenvalues in the gap. Theorem 4.3 shows that eigenfunctions of the approximating problem converge to eigenfunctions of the full (perturbed) problem and that multiplicity is conserved. Theorem 4.5 follows and with the aid of Lemma 4.6 we get rid of the approximating index $n$ in Estimate (4.5). Thus we have proven our main result Theorem 1.1.

\section{MANifOldS With SPECTRAL GAPS}

For the convenience of the reader, we cite the results on examples of manifolds with spectral gaps given in $[\mathrm{P} 2]$ and $[\mathrm{Y}]$. More details and further references can be found therein. 
Conformal periodic manifolds. Let $\mathcal{M}$ be a $\Gamma$-periodic manifold of dimension $d$ with periodic metric $g$. Furthermore, suppose that $\left(\rho_{\varepsilon}\right), \varepsilon>0$, is a family of smooth periodic functions $\rho_{\varepsilon}: \mathcal{M} \longrightarrow(0, \infty)$, i.e., $\rho_{\varepsilon}(\gamma x)=\rho_{\varepsilon}(x)$ for all $\gamma \in \Gamma$ and $x \in \mathcal{M}$. We denote by $\mathcal{M}_{\varepsilon}$ the manifold $\mathcal{M}$ with metric $g_{\varepsilon}:=\rho_{\varepsilon}^{2} g$. Similarly we define $M_{\varepsilon}$ for a period cell $M$. We have the following theorem:

Theorem 5.1. Suppose $d \geq 3$ and that $\rho_{\varepsilon}$ converges pointwise on $M$ to the indicator function of a closed set $X \subset \stackrel{\circ}{M}$ with smooth boundary. Then for each $k \in \mathbb{N}$, the $k$-th eigenvalue $\lambda_{k}\left(M_{\varepsilon}\right)$ with Dirichlet, Neumann or $\theta$-periodic boundary condition converge to $\lambda_{k}^{\mathrm{N}}(X)$ as $\varepsilon \rightarrow 0$ (uniformly in $\theta$ ). In particular, for all $n \in \mathbb{N}$ there exists $\varepsilon>0$ such that $M=M_{\varepsilon}$ satisfies the gap condition (1.1) for $k=1, \ldots, n$ (provided $\lambda_{k}^{\mathrm{N}}(X)$ is a simple eigenvalue).

The precise assumptions on $\rho_{\varepsilon}$ and $X$ resp. $M$ and the proof of this theorem in the $\theta$-periodic case can be found in $[\mathrm{P} 1]$ resp. [P2, Theorem 1.3]. There we also have presented an example for $d=2$. The proofs for the Dirichlet resp. Neumann case are similar.

Attaching small cylindrical ends. Let $X$ be a compact Riemannian manifold of dimension $d \geq 2$ with metric $g$. Let $x_{1}, x_{2} \in X$ be two distinct points. On $X \backslash\left\{x_{1}, x_{2}\right\}$ we change the metric such that a cylinder of radius $\varepsilon$ and length $\varepsilon / 2$ is isometrically embedded at each point $x_{i}$. Here, a cylinder is a product of the $(d-1)$-dimensional unit sphere $\mathbb{S}^{d-1}$ and an interval. We denote the (completion of the) resulting manifold by $M_{\varepsilon}$. Note that $\partial M_{\varepsilon}$ has two additional components $\partial_{1} M_{\varepsilon}$ and $\partial_{2} M_{\varepsilon}$ each of them being isometric to $\mathbb{S}^{d-1}$. A typical example of a period cell in the case when $X$ is a 2-dimensional torus is drawn in Figure 2 on page 25.

For $\gamma \in \mathbb{Z}$ let $\gamma M_{\varepsilon}$ be a copy of $M_{\varepsilon}$. Identifying $\partial_{2} \gamma M_{\varepsilon}$ with $\partial_{1}(\gamma+1) M_{\varepsilon}$ pointwise we obtain a $\mathbb{Z}$-periodic manifold $\mathcal{M}_{\varepsilon}$ with period cell $M_{\varepsilon}$. Similarly we can construct $\Gamma$-periodic manifolds by attaching $2 r$ cylindrical ends if $r$ denotes the number of generators of $\Gamma$. For a detailed construction we refer to [P2].

Note that the period cell $M_{\varepsilon}$ always has smooth boundary $\partial M$, i.e., we can allow non-compact perturbations in the next sections.

Intuitively, the period cell $M_{\varepsilon}$ is close to the original manifold $X$ if $\varepsilon$ is small. The next theorem shows that the same is true for the eigenvalues:

Theorem 5.2. Assume that the periodic manifold $\mathcal{M}_{\varepsilon}$ and the period cell $M_{\varepsilon}$ are constructed as above. Then for each $k \in \mathbb{N}$, the $k$-th eigenvalue $\lambda_{k}\left(M_{\varepsilon}\right)$ with Dirichlet, Neumann or $\theta$-periodic boundary condition converge to $\lambda_{k}(X)$ as $\varepsilon \rightarrow 0$ (uniformly in $\theta$ ). In particular, for all $n \in \mathbb{N}$ there exists $\varepsilon>0$ such that $M=M_{\varepsilon}$ satisfies the gap condition (1.1) for $k=1, \ldots, n$ (provided $\lambda_{k}(X)$ is a simple eigenvalue).

Again, the proof of this theorem and related results can be found in $\mathbb{P 2}$, Theorem 1.1]. Note that in Theorem 5.1 as well as in Theorem 5.2 the decoupling of the different period cells $\gamma M_{\varepsilon}$ is responsible for the gaps. 
Periodically curved quantum wave guides. Here, we present an example given by Yoshitomi [Y]. We consider a 2-dimensional planar strip $\mathcal{M}_{\varepsilon}$ obtained by sliding the normal segment of length $\varepsilon$ along a periodically curved path $\omega$. Note that the Dirichlet Laplacian on $M_{\varepsilon}$ is the Hamiltonian for an electron confined in a quantum wire on a planar substrate, where the vertical dimension is separated (cf. $[\mathrm{ES} \|$ ).

Suppose $\varepsilon_{0}>0$. Let $\kappa: \mathbb{R} \longrightarrow\left(-1 / \varepsilon_{0}, \infty\right)$ be a smooth and $2 \pi$-periodic map (i.e., $\kappa(s+2 \pi)=\kappa(s)$ for all $s \in \mathbb{R})$. Then

$$
\omega(s):=\left(x_{\cos }(s), x_{\sin }(s)\right) \quad \text { with } \quad x_{f}(s):=\int_{0}^{s} f\left(-\int_{0}^{s^{\prime}} \kappa\left(s^{\prime \prime}\right) \mathrm{d} s^{\prime \prime}\right) \mathrm{d} s^{\prime}
$$

for $f=\cos$ or $f=\sin$ is a curve in $\mathbb{R}^{2}$ with curvature $\kappa$. We denote by $\dot{\omega}^{\perp}(s):=$ $\left(-\dot{x}_{\sin }(s), \dot{x}_{\cos }(s)\right)$ the normal unit vector with respect to $\dot{\omega}(s)$. We set

$$
\mathcal{M}_{\varepsilon}:=\left\{\omega(s)+u \dot{\omega}^{\perp}(s) \mid s \in \mathbb{R}, 0 \leq 0 \leq \varepsilon\right\}
$$

and suppose that

$$
\Phi_{\varepsilon_{0}}: \mathbb{R} \times\left[0, \varepsilon_{0}\right] \longrightarrow \mathcal{M}_{\varepsilon}, \quad(s, u) \longmapsto \omega(s)+u \dot{\omega}^{\perp}(s)
$$

is a diffeomorphism (for the precise assumptions see [Y]). This diffeomorphism allows us to calculate the Dirichlet Laplacian on $\mathcal{M}_{\varepsilon}, 0<\varepsilon<\varepsilon_{0}$, in coordinates $(s, u)$. We furthermore assume that $\int_{0}^{2 \pi} \kappa(s) \mathrm{d} s=0$. Then $\omega$ is also $2 \pi$-periodic and $\mathcal{M}_{\varepsilon}$ is indeed a $\mathbb{Z}$-periodic manifold with a period cell $M_{\varepsilon}$ given by $\Phi_{\varepsilon}([0, \varepsilon] \times$ $[0,2 \pi])$. We set

$$
K:=-\frac{\mathrm{d}^{2}}{\mathrm{~d} s^{2}}-\frac{1}{4} \kappa^{2} .
$$

In $L_{2}([0,2 \pi])$ we regard the $\theta$-periodic realisation of $K$ and denote the corresponding eigenvalues by $\lambda_{k}^{\theta}(K)$. Yoshitomi proved that for all $k \in \mathbb{N}$,

$$
\lambda_{k}^{\theta}\left(M_{\varepsilon}\right)=\frac{\pi^{2}}{\varepsilon^{2}}+\lambda_{k}^{\theta}(K)+O(\varepsilon), \quad \varepsilon \rightarrow 0
$$

uniformly in $\theta$, where $\lambda_{k}^{\theta}\left(M_{\varepsilon}\right)$ denotes the eigenvalues of the Laplacian on $M_{\varepsilon}$ with Dirichlet boundary condition at $u=0$ and $u=\varepsilon$, and $\theta$-periodic boundary condition at $s=0$ and $s=2 \pi$. A similar identity holds for Dirichlet resp. Neumann boundary condition at $s=0$ and $s=2 \pi$ simultaneously for $\Delta_{M_{\varepsilon}}$ and $K$.

Furthermore, Yoshitomi proved that if $\kappa \neq 0$ there exists $k \in \mathbb{N}$ such that the $k$-th band and the $(k+1)$-st band of $\Delta_{\mathcal{M}_{\varepsilon}}^{\mathrm{D}}$ are disjoint. He uses classical results about the inverse problem for Hill's equation (cf. [GaTr]) to show that the periodic one-dimensional operator $K$ in $L_{2}(\mathbb{R})$ has a gap between the $k$-th and the $(k+1)$-st band. In our paper, we need the stronger assumption (1.1) asserted by the following theorem: 
Theorem 5.3. Assume in addition that the curvature $\kappa$ is even, i.e., $\kappa(-s)=$ $\kappa(s)$. Then for all $k \in \mathbb{N}$ the $k$-th band $B_{k}(K)$ of the periodic operator $K$ in $L_{2}(\mathbb{R})$ is given by

$$
B_{k}(K)=\left[\lambda_{k}^{\mathrm{D}}(K), \lambda_{k}^{\mathrm{N}}(K)\right]
$$

Here $\lambda_{k}^{\mathrm{D}}(K)$ resp. $\lambda_{k}^{\mathrm{N}}(K)$ denotes the operator $K$ in $L_{2}([0,2 \pi])$ with Dirichlet resp. Neumann boundary conditions. In particular if $\kappa \neq 0$ there exists $k \in \mathbb{N}$ such that the gap condition (1.1) is satisfied for the Dirichlet Laplacian on $\mathcal{M}_{\varepsilon}$ provided $\varepsilon$ is small enough.

Proof. Since $\kappa$ is even, $K$ has an even potential. In particular, (5.3) holds iff the potential is even (cf. GaTr). The rest follows from $[\mathrm{Y}$.

Yoshitomi also localized the gaps: he proved that the $k$-th gap of $K$ is open if the $k$-th Fourier coefficient of $\kappa^{2}$ is not 0 .

\section{EXAmples of PeRturbations}

In this section we discuss some examples of the great variety of possible perturbations. The only restriction is the knowledge of eigenvalue estimates of the perturbed and unperturbed problem to calculate the right hand sides of (1.6) and (1.7). Here, we always assume that $\mathcal{M}$ is a periodic manifold with periodic metric $g$ and period cell $M$ such that the gap condition (1.1) is fulfilled.

By the Weyl asymptotic distribution of eigenvalues (1.8) and by (1.9) we expect that an infinite number of eigenvalue branches comes from above crossing the level $\lambda$ if we increase the volume of $M_{\tau}^{m}$ to infinity. Therefore, we expect an infinite number of parameters $\tau$, such that $\lambda$ is an eigenvalue of $\Delta_{\mathcal{M}_{\tau}}$ by Theorem 1.1.

In contrast, if we shrink the volume of $M_{\tau}^{m}$, we would expect only a finite number of eigenvalues (depending on the volume of $M^{m}$ ). Here, we think of finitely many eigenvalue branches crossing the level $\lambda$ from below.

Conformal perturbations. First, we give examples of conformal perturabtions. Suppose that $\rho_{\tau}: \mathcal{M} \longrightarrow(0, \infty), \tau \geq 0$, is a family of smooth functions. The perturbed metric is given by $g_{\tau}=\rho_{\tau}^{2} g$. Clearly, if the family $\left(\rho_{\tau}\right)$ satisfies

$$
\begin{aligned}
\rho_{0}(x) & =1, & & x \in \mathcal{M} \\
\left\|\rho_{\tau}-\rho_{\tau_{0}}\right\|_{C^{1}} & \rightarrow 0 & & \text { on } \mathcal{M} \text { as } \tau \rightarrow \tau_{0}, \\
\rho_{\tau}(x) & =1, & & x \in R^{m}
\end{aligned}
$$

for all $\tau_{0} \geq 0$ and sufficiently large $m \in \mathbb{N}$ then $\left(g_{\tau}\right)$ satisfies Conditions (1.2), (1.3) and (1.4). Here, the $C^{1}$-norm of functions is defined in the usual way. Furthermore, if $\partial M$ is smooth we can also allow non-compact conformal perturbations which are small outside $M^{m}$, i.e.,

$$
\sup _{\tau \geq 0}\left\|\rho_{\tau}-1\right\|_{C^{1}} \rightarrow 0 \quad \text { on } R^{m} \text { as } m \rightarrow \infty
$$


If there is a non-compact perturbation we fix $m$ large enough such that Equation (1.6) resp. (1.7) holds.

Furthermore, we assume that the conformal factor is constant on some compact submanifold $K \subset M^{m}$ with non-empty interior and piecewise smooth boundary, i.e.,

$$
\rho_{\tau}(x)=c_{\tau}, \quad x \in K
$$

where $c_{\tau}>0$ is a constant. Again, $K_{\tau}$ denotes the manifold $K$ with metric $\rho_{\tau}^{2} g=c_{\tau}^{2} g$.

Eigenvalues coming from above. In our first example we blow up a subset of $M^{m}$ by conformal factors:

Proposition. Suppose that $\lambda \in I_{k}$ lies between the $k$-th and the $(k+1)$-st band and that $c_{\tau} \rightarrow \infty$ as $\tau \rightarrow \infty$. Then

$$
\mathcal{N}(\tau, \lambda) \geq \frac{\omega_{d}}{(2 \pi)^{d}} \lambda^{\frac{d}{2}} \operatorname{vol}\left(K_{\tau}\right)-k m-\delta(\tau)
$$

where $\delta(\tau) \searrow 0$ as $\tau \rightarrow \infty$. In particular, $\mathcal{N}(\tau, \lambda) \rightarrow \infty$ as $\tau \rightarrow \infty$, i.e., there exist an infinite number of parameters $\tau$ such that $\lambda$ is an eigenvalue of $\Delta_{\mathcal{M}_{\tau}}$.

Proof. Applying the Min-max principle we conclude

$$
\lambda_{j}^{\mathrm{D}}\left(M_{\tau}^{m}\right) \leq \lambda_{j}^{\mathrm{D}}\left(K_{\tau}\right)=c_{\tau}^{-2} \lambda_{j}^{\mathrm{D}}(K) \rightarrow 0
$$

as $\tau \rightarrow \infty$ since $\lambda_{j}^{\mathrm{D}}(K)>0$ for all $j \in \mathbb{N}$. Therefore all eigenvalue branches $\tau \mapsto \lambda_{j}^{\mathrm{D}}\left(M_{\tau}^{m}\right), j \in \mathbb{N}$, cross the level $\lambda$ from above (at least once). Furthermore, there exists a positive function $\delta(\tau) \rightarrow 0$ as $\tau \rightarrow \infty$ such that

$$
\operatorname{dim}_{\lambda}^{\mathrm{D}}\left(M_{\tau}^{m}\right) \geq \operatorname{dim}_{\lambda}^{\mathrm{D}}\left(K_{\tau}\right)=\operatorname{dim}_{c_{\tau}^{2} \lambda}^{\mathrm{D}}(K) \geq \frac{\omega_{d}}{(2 \pi)^{d}} \lambda^{\frac{d}{2}} c_{\tau}^{d} \operatorname{vol}(K)-\delta(\tau)
$$

by the Weyl asymptotic distribution (1.8). The result follows from Theorem 1.1 and Lemma 4.2.

Eigenvalues coming from below. Next we shrink the manifold $M^{m}$ on $K$ :

Proposition. Suppose that $\lambda \in I_{k}$ and that $c_{\tau} \rightarrow 0$ as $\tau \rightarrow \infty$. Then we have

$$
\mathcal{N}(\tau, \lambda) \geq k m-1
$$

for $\tau$ sufficiently large. In particular, there exist at least a finite number of parameters $\tau$ such that $\lambda$ is an eigenvalue of $\Delta_{\mathcal{M}_{\tau}}$.

Proof. The Min-max principle yields

$$
\lambda_{j}^{\mathrm{N}}\left(M_{\tau}^{m}\right) \geq \lambda_{j}^{\mathrm{N}}\left(K_{\tau}\right)=c_{\tau}^{-2} \lambda_{j}^{\mathrm{N}}(K) \rightarrow \infty
$$

as $\tau \rightarrow \infty$ for all $j \in \mathbb{N}$, except for the first Neumann eigenvalue $\lambda_{1}^{\mathrm{N}}(K)=0$. Therefore the eigenvalue branches $\tau \mapsto \lambda_{j}^{\mathrm{N}}\left(M_{\tau}^{m}\right), j \in \mathbb{N} \backslash\{1\}$ with $\lambda_{j}^{\mathrm{N}}\left(M_{0}^{m}\right)<\lambda$, cross the level $\lambda$ from below (at least once). Lemma 4.2 yields that there are exactly $k m-1$ such eigenvalue branches. Again we apply Theorem 1.1. 
Remark 6.1. If $d=2$ one has monotonically decreasing (increasing) eigenvalue branches $\tau \mapsto \lambda_{j}^{\mathrm{N}}\left(M_{\tau}^{m}\right)$ if the conformal factor $\rho_{\tau}$ is monotonically increasing (decreasing). Here, the conformal factor only occurs in the norm, not in the quadratic form. The monotonicity follows from the Min-max principle. This monotonicity can be a first step in the proof of an upper bound on the counting function $\mathcal{N}(\tau, \lambda)$ (see the introduction).

Finally, let us note the following theorem combining results of this section and of [P2] (cf. Theorem 5.1):

Theorem 6.2. Let $\mathcal{M}$ be a periodic Riemannian manifold of dimension $d \geq 3$ with metric $g$. For any $n \in \mathbb{N}$ there exists a periodic metric $g_{n}$ conformal to $g$ such that the corresponding Laplacian has at least $n$ spectral gaps. Furthermore, there exist (non-periodic) metrics on $\mathcal{M}$ conformal to $g$ such that the corresponding Laplacian has an eigenvalue in a gap of the essential spectrum.

\section{Diffeomorphic perturbations.}

Eigenvalues from above. Here, we show how perturbations $\widetilde{\mathcal{M}}_{\tau}$ diffeomorphic to $\mathcal{M}$ can be reduced to the case already treated. We think of $\widetilde{\mathcal{M}}_{\tau}$ as being a smooth deformation of $\mathcal{M}$. For example, consider a quantum wave guide $\mathcal{M}=\mathcal{M}_{\varepsilon}$ as defined in (5.1). We assume that the gap condition (1.1) holds for some fixed $\varepsilon>0$. Let the perturbation of $\mathcal{M}$ be given by cutting the strip at $s=0$ and inserting a "bubble" $K_{\tau}$ which blows up as $\tau$ increases.

To be more precise, suppose that $K_{\tau}$ is a simply connected closed subset of $\mathbb{R}^{2}$ such that $K_{\tau}$

- is a rectangle of length $\tau$ and width $\varepsilon$ for small $\tau$, in particular, $K_{0}$ has empty interior;

- contains a circle of radius $\tau$ or, alternatively, a rectangle of length $\tau$ and width $\varepsilon$ for all $\tau$ large enough;

- has two ends $A_{\tau, 1}$ and $A_{\tau, 2}$, each of them being isometric to a rectangle of width $\varepsilon$;

- depends continuously on $\tau$, i.e., the two boundary curves of $\partial K_{\tau} \backslash\left(A_{\tau, 1} \cup A_{\tau, 2}\right)$ are smooth and depend continuously on $\tau$ and the curve parameter.

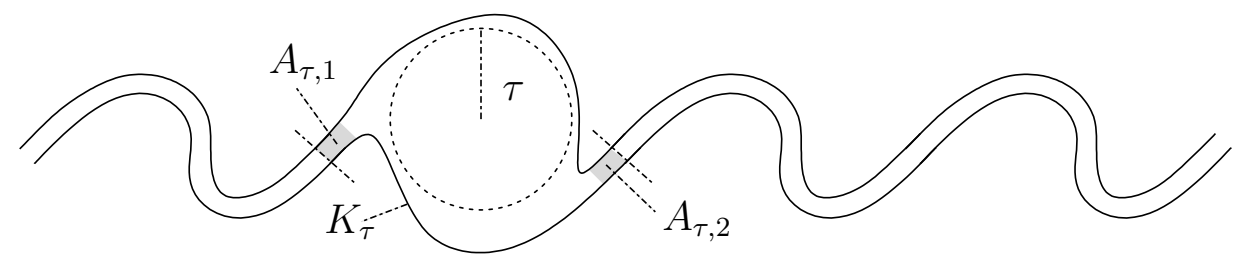

Figure 1. A quantum wave guide perturbed by inserting an increasing bubble.

We denote the intersected quantum wave guide together with the inserted bubble by $\widetilde{\mathcal{M}}_{\tau}$ (see Figure 1). Note that there exists a diffeomorphism $\Phi_{\tau}: \mathcal{M} \longrightarrow \widetilde{\mathcal{M}}_{\tau}$ 
such that $\Phi_{\tau}(x)=x$ outside a compact set (with the obvious identification of the two ends of the unperturbed and perturbed quantum wave guide).

The Euclidean metric $g$ on $\widetilde{\mathcal{M}}_{\tau}$ is pulled back on $\mathcal{M}$ via $\Phi_{\tau}$, i.e., $g_{\tau}:=\Phi_{\tau}^{*} g$. Note that $(\tau, x) \mapsto g_{\tau}(x)$ resp. $(\tau, x) \mapsto \partial_{i} g_{\tau}(x)$ are uniformly continuous maps on $\left[0, \tau_{0}\right] \times \mathcal{M}$ for all $\tau_{0}>0$ since $g_{\tau}=g$ outside a compact set and since $g$ is periodic, thus uniformly continuous. Therefore, it is easy to check that the Assumptions (1.2) to (1.4) are satisfied.

Proposition. Suppose that $\lambda \in I_{k}$ and that $\widetilde{\mathcal{M}}_{\tau}$ is the perturbed manifold $\mathcal{M}$ obtained by the above construction. Then we have $\mathcal{N}(\tau, \lambda) \rightarrow \infty$ as $\tau \rightarrow \infty$.

Proof. The proof is similar to the proof of Proposition 6. Note that in the case of an inserted rectangle of length $\tau$, we have $\lambda_{k}^{\mathrm{D}}\left(K_{\tau}\right) \leq(\pi k)^{2} / \tau^{2}$ for all $\tau \geq \tau_{0}$ where $\tau_{0}$ depends on $k$.

Remark 6.3. Note that the eigenvalue branches $\tau \mapsto \lambda_{j}^{\mathrm{N}}\left(K_{\tau}\right)$ are monotonically decreasing by the Min-max principle at least in the case when $K_{\tau}$ is isometric to a rectangle of length $\tau$ and width $\varepsilon$. This monotonicity can be a first step in the proof of an upper bound on the counting function $\mathcal{N}(\tau, \lambda)$ (see the introduction).

\section{TOpological PERTURBAtions}

Eigenvalues from below. In this final section we show how to deal with certain non-homeomorphic perturbations. We allow more general examples of perturbed Riemannian manifolds $\widetilde{\mathcal{M}}_{\tau}$ diffeomorphic to $\dot{\mathcal{M}}:=\mathcal{M} \backslash H$ where $H$ consists of discrete points. We think of $\widetilde{\mathcal{M}}_{\tau}$ being a smooth deformation of $\dot{\mathcal{M}}$. Note that the substraction of a discrete set of points has no influence on the Laplacian as a self-adjoint operator as we will see now.

Suppose that $X$ is a complete Riemannian manifold of dimension $d \geq 2$. Let $H \subset X \backslash \partial X$ be a discrete set of points. On $\dot{X}:=X \backslash H$ we define the Laplacian with Dirichlet resp. Neumann boundary condition on $\partial X$ as in Section 2. At $H$ we always assume a Dirichlet boundary condition, i.e., we start from the quadratic form $\check{q}_{X}$ defined for smooth functions with support away from $H$. Note that the Hilbert spaces $L_{2}(X)$ and $L_{2}(\dot{X})$ agree since $H$ has measure 0. Furthermore, the substraction of a discrete set of points $H$ has no effects on the Laplacian either (with any boundary condition):

Theorem 7.1. Suppose that $\partial X=\emptyset$. For every function $u \in \operatorname{dom} q_{X}$ there exists a sequence $\left(u_{n}\right)$ of smooth functions $u_{n} \in \operatorname{dom} q_{X}$ with support away from $H$ such that $u_{n} \rightarrow u$ in the form norm $\left(\|u\|_{X}^{2}+q_{X}(u)\right)^{\frac{1}{2}}$. In particular, dom $q_{\dot{X}}=\operatorname{dom} q_{X}$ and therefore, the corresponding operators agree as operators in $L_{2}(X)$.

If $\partial X \neq \emptyset$ the same is true for the quadratic form with Dirichlet resp. Neumann (or any other) boundary condition on $\partial X$. Again, the corresponding Laplacians on the dotted and undotted manifolds agree. 
Proof. For a single point see $\mathbb{C F}$, Lemma 1]. Clearly we can generalise this result to a discrete set of points $H$. Since we have defined the Laplacian via quadratic forms the equality of the operators follows.

We consider the following example: suppose that we bore a hole around each point of $H$ in the periodic manifold $\mathcal{M}$. For simplicity, we assume that $H$ consists of $m$ points $\gamma x_{0}$ where $\gamma \in \Gamma^{m}$ (recall that $\Gamma^{m}$ is an exhausting sequence of $\Gamma$ as defined in the beginning) and $x_{0} \in M$ with $\operatorname{dist}\left(x_{0}, \partial M\right)<\varepsilon_{0} / 2$. Here, $M$ denotes a fixed period cell of $\mathcal{M}$ for which the gap condition (1.1) holds and $\varepsilon_{0}>0$ is the injectivity radius of $\mathcal{M}$, i.e., the exponential map is defined on all balls of radius smaller than $\varepsilon_{0}$.

To define properly the smooth dependence of the boundary on the parameter $\tau$, we need the following construction: let $f: \mathcal{M} \longrightarrow[0, \infty]$ be a continuous map. We think of $f$ quantifying the level of perturbation: $f(x)=0$ resp. $f(x)=\infty$ means that $x \in \mathcal{M}$ is always resp. never affected by the perturbation. In particular, we assume

- that $f(x)=\operatorname{dist}\left(x, \gamma x_{0}\right)$ for all $x \in \gamma M \operatorname{such}$ that $\operatorname{dist}\left(x, \gamma x_{0}\right)<\varepsilon_{0} / 2$, in particular $f\left(\gamma x_{0}\right)=0$ for all $\gamma \in \Gamma^{m}$;

- that $f(x)=\infty$ for all $x \in R^{m}=\left(\Gamma \backslash \Gamma^{m}\right) M$ and all $x \in \Gamma^{m} \partial M$, i.e., the union of all translates $\gamma \partial M, \gamma \in \Gamma^{m}$;

- that $f$ is smooth on $\{x \in \mathcal{M} \mid 0<f(x)<\infty\}$ and that all $\tau \in(0, \infty)$ are regular values of $f$, i.e., if $f(x)=\tau$ then $\mathrm{d} f_{x} \neq 0$;

- that $Z:=\left\{x \in M^{m}=\Gamma^{m} M \mid f(x)=\infty\right\}$ is the finite union of compact smooth submanifolds $Z_{i}$ of dimension $d_{i}<d$ with piecewise smooth boundary such that $Z_{i} \cap Z_{j}$ is either empty or of dimension smaller than $d_{i}$ and $d_{j}$ (cf. Figure 2);

- that $\left\{x \in M^{m} \mid f(x)>\tau\right\}$ is contained in the $1 / \tau$-neighbourhood of $Z$, i.e., $\operatorname{dist}(x, Z)<1 / \tau$ for all $x \in M^{m}$ with $f(x)>\tau$.

Let

$$
\begin{aligned}
\widetilde{\mathcal{M}} & :=\{(\tau, x) \in[0, \infty) \times \mathcal{M} \mid f(x)>\tau\} \quad \text { resp. } \\
Y & :=\{(\tau, x) \in[0, \infty) \times \mathcal{M} \mid f(x)=\tau\}
\end{aligned}
$$

be the fibred manifold with fibres

$$
\begin{aligned}
\widetilde{\mathcal{M}}_{\tau} & :=\{x \in \mathcal{M} \mid f(x)>\tau\} \quad \text { resp. } \\
Y_{\tau} & :=\{x \in \mathcal{M} \mid f(x)=\tau\} .
\end{aligned}
$$

We consider these manifolds as submanifolds of the product of the Riemannian manifolds $[0, \infty)$ and $\mathcal{M}$ (with the induced metrics). Note that $\mathcal{M} \backslash \widetilde{\mathcal{M}}_{\tau}$ is a manifold diffemorphic to $m$ copies of a closed ball in $\mathbb{R}^{d}$ with smooth boundary $Y_{\tau}$ diffeomorphic to $m$ copies of the sphere $\mathbb{S}^{d-1}$ provided $0<\tau<\infty$. Furthermore, note that $Y$ consists of $m$ cones. In a neighbourhood of (each component of) $Y$ 


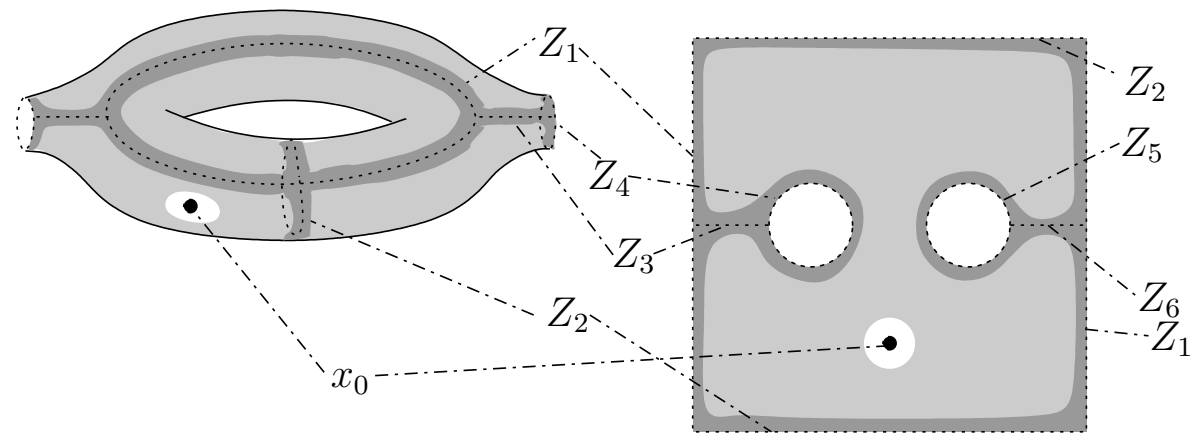

Figure 2. A period cell with the dotted set $Z$ and its smooth components $Z_{i}$. On the right hand side one has to identify opposite sides of the square. Note that the complement of $Z$ in the period cell is always homeomorphic to an open ball in $\mathbb{R}^{d}$. The (light and dark) grey set is the perturbed manifold for small $\tau>0$, the dark grey set is the perturbed manifold for large $\tau$.

we introduce normal (or Fermi) coordinates given by a chart $\varphi: U \longrightarrow V$ where

$$
U=\left\{(\tau, x) \in \widetilde{\mathcal{M}} \mid 0<\operatorname{dist}(x, Y)<r_{0}(\tau)\right\}
$$

(see e.g. [Sp, p. 9-59-9-62]). Here, the function $r_{0}$ is supposed to be smooth. Then the image of the chart is given by

$$
V=\left\{(s, \tau, y) \in(0, \infty) \times[0, \infty) \times Y \mid s<r_{0}(\tau)\right\} .
$$

In particular, for fixed $\tau$ we have a chart $\varphi_{\tau}: U_{\tau} \longrightarrow V_{\tau}$ for the corresponding fibres.

Note that $\widetilde{\mathcal{M}}$ is diffeomorphic to $[0, \infty) \times \dot{\mathcal{M}}$. We construct the diffeomorphism explicitly. Let

$$
r:[0, \infty) \times[0, \infty) \longrightarrow[0, \infty)
$$

be a continuous map (smooth on the interior) with bounded derivatives such that

$$
r_{\tau}:=r(\tau, \cdot):[0, \infty) \longrightarrow[\tau, \infty)
$$

is bijective and

$$
r_{\tau}(0)=\tau \quad \text { and } \quad r_{\tau}(s)=s, \quad s \geq r_{0}(\tau) / 2 .
$$

Then

$$
\Phi:[0, \infty) \times \dot{\mathcal{M}} \longrightarrow \widetilde{\mathcal{M}}, \quad \Phi(\tau, x):=\varphi^{-1}\left(r_{\tau}(s), \tau, y\right)
$$

with $(s, y):=\varphi_{\tau}(x)$ is a diffeomorphism. Clearly, the corresponding maps on the fibres $\Phi_{\tau}: \dot{\mathcal{M}} \longrightarrow \widetilde{\mathcal{M}}_{\tau}$ are also diffeomorphisms. For a technical reason we do not use $\dot{\mathcal{M}}$ but

$$
\hat{\mathcal{M}}:=\left\{x \in \mathcal{M} \mid \operatorname{dist}(x, H)>\varepsilon_{0} / 2\right\}
$$


as a reference manifold. Note that there exists a diffeomorphism

$$
\psi: \hat{\mathcal{M}} \longrightarrow \dot{\mathcal{M}}
$$

Therefore, $\hat{\mathcal{M}}$ is also diffeomorphic to each fibre $\widetilde{\mathcal{M}}_{\tau}$ of $\widetilde{\mathcal{M}}$. The technical reason is that we need an analogue to Theorem 3.5 where we have used regularity theory. On $\dot{\mathcal{M}}$ we would have coefficients which cannot be continuously extended onto $H$. Blowing up the holes and pulling back the metrics avoids this difficulty. Therefore, we have a diffeomorphism

$$
\Psi:[0, \infty) \times \hat{\mathcal{M}} \longrightarrow \widetilde{\mathcal{M}}, \quad \Psi(\tau, x):=\left(\tau, \Phi_{\tau}(\psi(x))\right) .
$$

There is a natural metric on $\widetilde{\mathcal{M}}$, namely $\widetilde{h}:=\mathrm{d} \tau^{2}+g$ where $g$ is the (restriction of the) periodic metric on $\mathcal{M}$. Let $h:=\Psi^{*} \widetilde{h}$ be the pull-back of the metric $\widetilde{h}$. Note that $h$ (and its derivatives) are uniformly continuous maps on $\left[0, \tau_{0}\right] \times$ $\hat{\mathcal{M}}$. Therefore, the restriction $g_{\tau}$ of $h$ onto the fibre $\hat{\mathcal{M}}$ satisfies the continuity property (1.3). Furthermore, Condition (1.4) is fulfilled.

Finally, replacing $\mathcal{M}$ by $\hat{\mathcal{M}}$ and $\Gamma^{\prime} M$ by $\Gamma^{\prime} M \cap \hat{\mathcal{M}}$ with metric $\psi^{*} g$ one can verify that all our results of Sections 3 and 4 remain true in virtue of Theorem 7.1 .

Therefore, we have the following result:

Proposition. Suppose that $\lambda \in I_{k}$. Let $\left(\widetilde{\mathcal{M}}_{\tau}\right)$ be the family of manifolds constructed above by removing closed sets from $M^{m}$. Then we have $\widetilde{\mathcal{N}}(\tau, \lambda) \geq k m$ for $\tau$ sufficiently large. Here, $\tilde{\mathcal{N}}(\tau, \lambda)$ denotes the counting function for the manifold $\widetilde{\mathcal{M}}_{\tau}$ defined as in (1.5). In particular, there exist a finite number of parameters $\tau$ such that $\lambda$ is an eigenvalue of $\Delta_{\widetilde{\mathcal{M}}_{\tau}}$.

Proof. The proof is the same as the proof of Proposition 6. Set $\widetilde{M}_{\tau}^{m}:=\widetilde{\mathcal{M}}_{\tau} \cap M^{m}$. We need the fact that $\lambda_{j}^{\mathrm{N}}\left(\widetilde{M}_{\tau}^{m}\right) \rightarrow \infty$ as $\tau \rightarrow \infty$ for all $j \in \mathbb{N}$ when $\operatorname{vol}\left(\widetilde{M}_{\tau}^{m}\right) \rightarrow 0$ as $\tau \rightarrow \infty$. This will be shown in the next lemma.

Remark 7.2. Note that we have imposed Neumann boundary condition only at the $\tau$-independent component $\partial M^{m}=\partial \widetilde{M}_{\tau}^{m}$ and Dirichlet boundary condition at $Y_{\tau}$. If there was only Neumann boundary condition the first eigenvalue would be 0 .

Remark 7.3. In this example, the eigenvalue branches $\tau \mapsto \lambda_{j}^{\mathrm{N}}\left(\widetilde{M}_{\tau}^{m}\right)$ are monotonically increasing due to the Min-max principle. This monotonicity can be a first step in the proof of an upper bound on the counting function $\mathcal{N}(\tau, \lambda)$ (see the introduction).

In the next lemma we show that the eigenvalues grow up to infinity when shrinking the volume of a manifold to 0 .

Lemma 7.4. Suppose that $K$ is a d-dimensional manifold with piecewise smooth boundary $\partial K$. Denote by $\left(K_{\tau}\right)$ a family of d-dimensional closed submanifolds of 
$K$ such that $\operatorname{vol} K_{\tau} \rightarrow 0$ as $\tau \rightarrow \infty$ and such that $\partial K_{\tau}$ depends smoothly on $\tau$ (defined in the same way as before). Furthermore, assume that $\partial K_{\tau}$ consists of two disjoint components $Y_{\tau}$ and $\partial K$ where $Y_{\tau}$ is smooth. Besides, suppose that

$$
K_{\tau} \subset\left\{x \in K \mid \operatorname{dist}(x, Z)<\frac{1}{\tau}\right\}:=N_{\tau}
$$

for $\tau$ large. Here, we assume that $Z$ is the finite union of compact smooth submanifolds $Z_{i}$ of dimension $d_{i}<d$ with piecewise smooth boundary such that $Z_{i} \cap Z_{j}$ is either empty or of dimension smaller than $d_{i}$ and $d_{j}$ (cf. Figure Q). Finally, suppose that $\partial K \subset Z$. Then $\lambda_{k}^{\mathrm{D}, \mathrm{N}}\left(K_{\tau}\right) \rightarrow \infty$ as $\tau \rightarrow \infty$ for all $k \in \mathbb{N}$ where we have imposed Dirichlet boundary conditions on $Y_{\tau}$ and Neumann boundary condition on $\partial K$.

Proof. We define the following isoperimetric (or Cheeger's) constant

$$
h_{\partial K}\left(K_{\tau}\right):=\inf _{\Omega_{\tau}} \frac{\operatorname{vol}_{d-1}\left(\partial \Omega_{\tau} \backslash \partial K\right)}{\operatorname{vol}_{d}\left(\Omega_{\tau}\right)}
$$

where $\Omega_{\tau}$ ranges over all open subsets of $K_{\tau}$ such that $\partial \Omega_{\tau} \backslash \partial K$ is smooth and $\partial \Omega_{\tau} \cap Y_{\tau}=\emptyset$. The subscript $\partial K$ indicates the subset of $\partial K_{\tau}$ where we have imposed Neumann boundary conditions. When measuring the surface we exclude the Neumann boundary part $\partial K$. If there were no Dirichlet boundary condition (i.e., $\partial K_{\tau}=\partial K$ ) we could choose $\Omega_{\tau}=K_{\tau}$. Therefore, $\partial \Omega_{\tau} \backslash \partial K=\emptyset$, i.e., $h_{\partial K}\left(K_{\tau}\right)=0$.

In the same way as in [C1, Chapter IV] we can prove the following estimate originally due to Cheeger:

$$
\lambda_{1}^{\mathrm{D}, \mathrm{N}}\left(K_{\tau}\right) \geq \frac{1}{4} h_{\partial K}\left(K_{\tau}\right)^{2} .
$$

It remains to show that $h_{\partial K}\left(K_{\tau}\right) \rightarrow \infty$ as $\tau \rightarrow \infty$. To this end let $\Omega_{\tau}$ be one of the sets taken in the definition of $h_{\partial K}\left(K_{\tau}\right)$. Since the set $Z$ is the finite union of smooth submanifolds $Z_{i}$ we decompose the (subset of a) tubular neighbourhood $N_{\tau}$ of $Z$ into a finite number of disjoint open sets $N_{\tau, i}$ such that $Z_{i}=N_{\tau, i} \cap Z$ and such that $N_{\tau} \backslash \bigcup_{i} N_{\tau, i}$ has $d$-dimensional volume 0 . If $d_{i}=d-1$ the set $N_{\tau, i}$ could lie on both sides of $Z_{i}$ (cf. Figure 3). In this case we decompose $N_{\tau, i}$ into its two components of $N_{\tau, i} \backslash Z_{i}$ (again denoted by $N_{\tau, i}$ ).

We set $A_{\tau, i}:=\left(\partial \Omega_{\tau} \cap N_{\tau, i}\right)$ and $\Omega_{\tau, i}:=\Omega_{\tau} \cap N_{\tau, i}$. With regard to the next lemma, we have

$$
\operatorname{vol}_{d-1}\left(\partial \Omega_{\tau} \backslash \partial K\right) \geq \sum_{i} \operatorname{vol}_{d-1}\left(A_{\tau, i}\right) \geq c \tau \sum_{i} \operatorname{vol}_{d}\left(\Omega_{\tau, i}\right)=c \tau \operatorname{vol}_{d}\left(\Omega_{\tau}\right)
$$

and we are done.

We still need the following final technical lemma which roughly says that the "local" isoperimetric constant in a small strip tends to infinity when the width of the strip tends to 0 . 


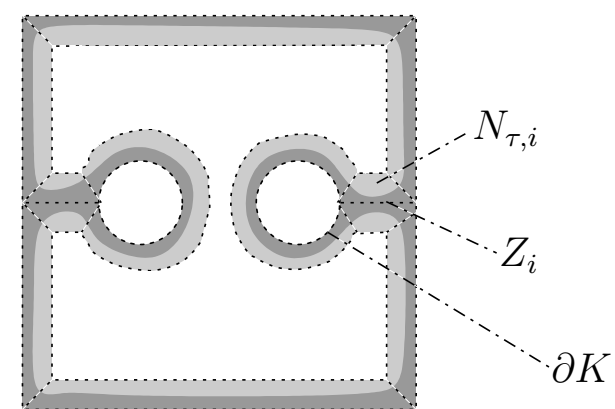

Figure 3. The decomposition of the tubular neighbourhood of $Z$. Again, we have to identify opposite sides of the square. Here, $K$ is the torus with two open balls cut out, $K_{\tau}$ is marked in dark grey and the tubular neighbourhood $N_{\tau}$ of radius $1 / \tau$ is marked in light and dark grey. Note that near $Z_{i}$, we have to decompose the tubular neighbourhood once more into two sets.

Lemma 7.5. With the notation from above we have

$$
\operatorname{vol}_{d-1}\left(A_{\tau, i}\right) \geq c \tau \operatorname{vol}_{d}\left(\Omega_{\tau, i}\right)
$$

where the constant $c$ only depends on the metric near $Z$.

Proof. Since $Z_{i}$ is a smooth submanifold of $K$ we can introduce normal coordinates $(y, z)$ on the tubular neighbourhood $N_{\tau, i}$ of $Z_{i}$ provided $\tau$ is large enough, i.e., $N_{\tau, i}$ is diffeomorphic to $B_{\tau} \times\left(Z_{i} \backslash \partial Z_{i}\right)$ where $B_{\tau}:=\left\{y \in \mathbb{R}^{d-d_{i}}|| y \mid<1 / \tau\right\}$ (resp. $B_{\tau}:=(0,1 / \tau)$ if $d_{i}=d-1$ ), see e.g. [Sp, p. 9-59-9-62]. Since $Z_{i}$ is compact and since the metric on $N_{\tau, i}$ depends continuously on the coordinates and smoothly on $\tau$ it suffices to prove the assertion in the case when the metric (in normal coordinates) has product structure $\mathrm{d} y^{2}+h$ where $h$ is the metric on $Z_{i}$.

Denote by $P_{\tau, i}$ the image of $A_{\tau, i}$ under the orthogonal projection onto $Z_{i}$. If we parametrize the smooth submanifold $A_{\tau, i}$ we can show that

$$
\operatorname{vol}_{d-1}\left(A_{\tau, i}\right) \geq c^{\prime} \tau^{-\left(d-1-d_{i}\right)} \operatorname{vol}_{d_{i}}\left(P_{\tau, i}\right)
$$

where the constant $c^{\prime}$ depends only on the metric $h$. Finally,

$$
\operatorname{vol}_{d}\left(\Omega_{\tau, i}\right) \leq \operatorname{vol}_{d}\left(N_{\tau, i}\right) \leq c^{\prime \prime} \tau^{-\left(d-d_{i}\right)} \operatorname{vol}_{d_{i}}\left(P_{\tau, i}\right)
$$

where $c^{\prime \prime}$ depends only on $d-d_{i}$. Therefore we have finished our proof.

\section{ACKNOWLEDGements}

I would like to thank Rainer Hempel for helpful discussions leading to this article. Furthermore I would like to thank Wolf Jung for his suggestions concerning the proofs of Lemmas 7.4 and 7.5 . 


\section{REFERENCES}

[AADH] S. Alama, M. Avellaneda, P.A. Deift, and R. Hempel, On the existence of eigenvalues of a divergence-form operator $A+\lambda B$ in a gap of $\sigma(A)$, Asymptotic Anal. 8 (1994), 311-344.

[ADH] S. Alama, P. A. Deift, and R. Hempel, Eigenvalue branches of the Schrödinger operator $H-\lambda W$ in a gap of $\sigma(H)$, Commun. Math. Phys. 121 (1989), no. 2, 291-321.

[Bi] M. Sh. Birman, Discrete spectrum of the periodic Schrödinger operator perturbed by a decaying potential, St. Petersbg. Math. J. 8 (1997), 1-14.

[BJR] O. Bratteli, P. E. T. Jørgensen, and D. W. Robinson, Spectral asymptotics of periodic elliptic operators, Math. Z. 232 (1999), no. 4, 621-650.

[BrS] J. Brüning and T. Sunada, On the spectrum of periodic elliptic operators, Nagoya Math. J. 126 (1992), 159-171.

[CF] I. Chavel and E. A. Feldman, Spectra of domains in compact manifolds, J. Funct. Anal. 30 (1978), 198-222.

[C1] I. Chavel, Eigenvalues in Riemannian geometry, Academic Press, Orlando, 1984.

[C2] , Riemannian geometry, Cambridge University Press, Cambridge, 1993.

[Da] E. B. Davies, Spectral theory and differential operators, Cambridge University Press, Cambridge, 1996.

[DH] P. A. Deift and R. Hempel, On the existence of eigenvalues of the Schrödinger operator $H-\lambda W$ in a gap of $\sigma(H)$, Commun. Math. Phys. 103 (1986), 461-490.

[DoL] H. Donnelly and P. Li, Pure point spectrum and negative curvature for noncompact manifolds, Duke Math. J. 46 (1979), 497-503.

[Do1] H. Donnelly, On $L^{2}-$ Betti numbers for Abelian groups, Can. Math. Bull. 24 (1981), 91-95.

[Do2] - Asymptotic properties of eigenfunctions - the hyperbolic plane, J. Anal. Math. 58 (1992), 135-151.

[EŠ] P. Exner and P. Šeba, Bound states in curved quantum waveguides, J. Math. Phys. 30 (1989), no. 11, 2574-2580.

[FH] R. Froese and I. Herbst, Realizing holonomic constraints in classical and quantum mechanics, Studies in Advanced Mathematics 16 (2000), 121-131.

[GiT] D. Gilbarg and N. S. Trudinger, Elliptic partial differential equations of second order, Springer-Verlag, Berlin, 1977.

[GaTr] J. Garnett and E. Trubowitz, Gaps and bands of one dimensional periodic Schrödinger operators II, Comment. Math. Helv. 62 (1987), 18-37.

[HB] R. Hempel and A. Besch, Magnetic barriers of compact support and eigenvalues in spectral gaps, Preprint (2000).

[He] E. Hebey, Sobolev spaces on Riemannian manifolds, Lecture Notes in Mathematics. Vol. 1635, Springer-Verlag, Berlin, 1996.

[Hi] P. D. Hislop, The geometry and spectra of hyperbolic manifolds, Proc. Indian Acad. Sci. Math. Sci. 104 (1994), 715-776, Spectral and inverse spectral theory (Bangalore, 1993).

[K] T. Kato, Perturbation theory for linear operators, Springer-Verlag, Berlin, 1966.

[Li] J. Li, The problem of eigenvalue on noncompact complete Riemannian manifold, J. Partial Differ. Equations 5 (1992), no. 4, 87-95.

[M] K. A Mitchell, Gauge fields and extrapotentials in constrained quantum systems, Phys. Rev. A 63 (2001), Nr. 042112.

[P1] O. Post, Periodic manifolds, spectral gaps, and eigenvalues in gaps, Ph.D. thesis, Technische Universität Braunschweig, 2000.

[P2] _ Periodic manifolds with spectral gaps, to appear in J. Diff. Equations. 
[RS1] M. Reed and B. Simon, Methods of modern mathematical physics I: Functional analysis, Academic Press, New York, 1980.

[RS4] M. Reed and B. Simon, Methods of modern mathematical physics IV: Analysis of operators, Academic Press, New York, 1978.

[Sa] O. L. Safronov, The discrete spectrum in the spectral gaps of semibounded operators with non-sign-definite perturbations, J. Math. Anal. Appl. 260 (2001), 641-652.

[Sp] M. Spivak, A comprehensive introduction to differential geometry, vol. 1, Publish or Perish, Boston, 1970.

[SV] Y. Safarov and D. Vassiliev, The asymptotic distribution of eigenvalues of partial differential operators, Translations of Mathematical Monographs 155, Providence, 1998.

[Y] K. Yoshitomi, Band gap of the spectrum in periodically curved quantum waveguides, J. Diff. Equations 142 (1998), 123-166.

Institut für Reine Und Angewandte Mathematik, Rheinisch-Westfälische Technische Hochschule Aachen, Templergraben 55, 52062 Aachen, Germany

E-mail address: post@iram.rwth-aachen.de 